\title{
Three-dimensional organization of dendrites and local axon collaterals of shell and core medium-sized spiny projection neurons of the rat nucleus accumbens
}

\author{
Yvette C. van Dongen - Philippe Mailly • \\ Anne-Marie Thierry · Henk J. Groenewegen · \\ Jean-Michel Deniau
}

Received: 15 October 2007 / Accepted: 15 January 2008/Published online: 1 February 2008

(C) The Author(s) 2008

\begin{abstract}
Medium-sized spiny projection neurons (MSN) in the head of the primate caudate nucleus are thought to have preferred dendritic orientations that tend to parallel the orientations of the striosomes. Moreover, recurrent axon collaterals of MSN in the rat dorsal striatum have been categorized into two types, i.e., restricted and widespread. The nucleus accumbens (Acb) has a highly complex compartmental organization, and the spatial organization of dendritic and axonal arbors of MSN has not yet been systematically studied. In this study, using singlecell juxtacellular labeling with neurobiotin as well as anterograde neuroanatomical tracing with biotinylated dextran amine, we investigated the three-dimensional (3D) organization of dendrites and axons of MSN of the rat Acb in relation to subregional (shell-core) and compartmental (patch-matrix) boundaries. Our results show that dendritic arbors of MSN in both the Acb shell and core subregions are preferentially oriented, i.e., they are flattened in at least one of the 3D-planes. The preferred orientations are influenced by shell-core and patch-matrix boundaries,
\end{abstract}

Y. C. van Dongen · H. J. Groenewegen ( $\square)$

Department of Anatomy and Neurosciences, Amsterdam, Research Institute Neurosciences Vrije Universiteit,

VU University Medical Center, P.O. Box 7057, MF-G102, 1007 MC Amsterdam, The Netherlands

e-mail: hj.groenewegen@vumc.nl

P. Mailly · A.-M. Thierry · J.-M. Deniau

Institut National de la Santé et de la Recherche Médicale, Unité 114, Chaire de Neuropharmacologie, Collège de France,

75231 Paris, France

P. Mailly

Neurobiologie des Signaux Intercellulaires, Centre National de la Recherche Scientifique, Université Pierre et Marie Curie, 75005 Paris, France suggesting parallel and independent processing of information. Dendritic orientations of MSN of the Acb core are more heterogeneous than those of the shell and the dorsal striatum, suggesting a more complex distribution of striatal inputs within the core. Although dendrites respect the shellcore and patch-matrix boundaries, recurrent axon collaterals may cross these boundaries. Finally, different degrees of overlap between dendritic and axonal arborizations of individual MSN were identified, suggesting various possibilities of lateral inhibitory interactions within and between, functionally distinct territories of the Acb.

Keywords Ventral striatum - Juxtacellular labeling .

Three-dimensional models · Medium-sized spiny projection neurons · Dendritic orientations .

Local axon collaterals

\section{Introduction}

The spatial arrangement of the dendrites of a neuron is an important feature of its specific ability to process incoming information, ultimately leading to an output of that neuron. For example, large pyramidal neurons in the cerebral cortex have a very specific and highly ordered threedimensional (3D) morphology of their dendrites and intracortical axons that is related to both the horizontal cortical lamination and the vertical columnar organization and, in this way, with specific cortical input-output features (Ramón y Cajal 1911; Gray 1959; Feldman 1984; DeFelipe et al. 2002). The striatum, the main input nucleus of the basal ganglia, lacks an apparent cytoarchitectonic feature like the cortical lamination and has a rather homogeneous cytoarchitectonic structure. Moreover, at first sight, the dendrites of its principal projection neurons, 
i.e., the medium-sized spiny projection neurons (MSN) appear to have a much less clearly ordered geometry than the cortical pyramidal neurons. However, Walker et al. (1993) described a preferred orientation of the dendritic arbors of MSN in the primate striatum along a rostraldorsal-medial to caudal-ventral-lateral axis. These authors suggested that this orientation tends to parallel the preferred orientation of the striatal compartments, i.e., the striosomes, in the primate striatum. Thus, the geometry of striatal MSN might, at least to some extent, be related to the striatal compartmental structure.

The ventral striatum is known to differ in its compartmental organization from the dorsal striatum. On the basis of the patterns of neurohistochemical staining, the nucleus accumbens (Acb), which is the major component of the ventral striatum, can be divided into a peripheral 'shell' and a central 'core' subregion. Within these subregions different smaller compartments exist (Zaborszky et al. 1985; Voorn et al. 1989; Zahm and Brog 1992; JongenRêlo et al. 1993, 1994). In view of the differences in compartmental structure between the dorsal and ventral striatum, a question is whether the dendrites of ventral striatal MSN have preferred orientations and whether the geometry of the dendrites of these neurons is in any way related to the orientation of the shell-core border or compartmental structure of the ventral striatum.

The MSN, in addition to innervating structures extrinsic to the striatum, have a local axon collateral network that arborizes within the striatum. Most recently, these local axon collaterals have been demonstrated to play an important role in GABA-mediated synaptic transmission between striatal spiny projection neurons (Tunstall et al. 2002; Czubayko and Plenz 2002; Taverna et al. 2004; Venance et al. 2004). These findings prompted us to analyze and describe the spatial organization of the recurrent axon collateral network of MSN. Kawaguchi et al. (1990) described two types of MSN in the rat dorsal striatum, one with local axon collaterals restricted to the dendritic field of the parent cell, and one with more widespread axon collaterals. The first type might be important for interactions between neurons within a particular functional striatal 'territory'. The second type might provide a possibility for more distant interactions, i.e., between functionally different 'territories'. The patterns of distribution of recurrent axon collaterals of MSN in relation to the parent cell body and the geometry of its dendritic arborizations, therefore, are two basic aspects of functional architecture of the striatum. Until now, very little is known about such intrinsic relationships within the ventral striatum.

In the present study, we examined the 3D organization of the dendrites and local axon collaterals of MSN in the rat Acb shell and core subregions, by using single-cell juxtacellular filling with neurobiotin (Pinault 1996) as well as anterograde neuroanatomical tracing with biotinylated dextran amine (Veenman et al. 1992). The geometrical aspects of the dendrites and recurrent axon collaterals of the injected neurons were studied in relation to the shell-core boundary and immunohistochemically defined compartments.

\section{Experimental procedures ${ }^{1}$}

Single-cell juxtacellular injections

\section{Animals}

Juxtacellular injection experiments were performed on 15 adult male Sprague Dawley (IFFA CREDO, Les Oncins, France) weighing 250-380 g. Surgical procedures were applied in strict accordance with the European Communities Council Directive 86/609/EEC (1986). The number of animals and their suffering was kept as low as possible.

\section{Animal preparation}

Animals were anesthetized by an injection of pentobarbital ( $1 \mathrm{ml} / \mathrm{kg}$, i.p.; Sanofi, Libourne, France) and fixed in a conventional stereotaxic frame. Anesthesia was maintained throughout the experiment by ketamine $(0.5 \mathrm{ml} / \mathrm{kg}$, i.m.; Imalgène 500, Rhone-Mérieux, France). In addition, incision and pressure points were infused with lidocaine. Throughout the experiment, heart beat and pedal withdrawal reflexes were monitored to assess the depth of anesthesia, and the body temperature was maintained between 37 and $38^{\circ} \mathrm{C}$ by the use of a homeothermic mat. Subsequently, the brain was exposed through small burr holes in the skull.

\section{Electrophysiological characterization of Acb neurons}

To label Acb neurons, electrophysiological responses of Acb cells evoked by electrical stimulation of either the ventral hippocampus (in particular the CA1 region) or the medial prefrontal cortex (PFC) were used as a guide for stereotaxic placement of recordings within the Acb shell or core, respectively. Stimulations $(200 \mu \mathrm{s}$ duration, 20-100 $\mu \mathrm{A}$ intensity) were applied at a depth of $7 \mathrm{~mm}$ from the cortical surface for CA1 (ipsilaterally to the

\footnotetext{
1 The anterograde tracer injections and the single-cell juxtacellular injections were performed in two different laboratories; minor differences in the surgical and staining procedures are the result of different traditions in these laboratories, rather than that they represent as essentially different steps in the protocols.
} 
recorded Acb) and $3 \mathrm{~mm}$ for the medial PFC (ipsilaterally or contralaterally to the recorded Acb). Stereotaxic coordinates of recording and stimulating sites were determined using the atlas of Paxinos and Watson (1986).

Extracellular single unit recordings were made in the Acb using glass pipettes (15-20 M $\Omega$ ) containing 1.5\% neurobiotin (Nb; Vector, Burlingame, CA, USA) in $0.5 \mathrm{M}$ $\mathrm{NaCl}$. The hippocampus and PFC were stimulated using a coaxial stainless steel electrode (diameter $400 \mu \mathrm{m}$; tipbarrel distance $300 \mu \mathrm{m}$ ). Action potentials of single neurons were recorded using the active bridge mode of an Axoclamp 2 B amplifier (Axon Instruments, Foster City, CA, USA), amplified $(1,000 \times)$, filtered $(0.3-3 \mathrm{kHz})$ with an AC/DC amplifier (DAM 50, World Precision Instruments, Hertfordshire, UK), and viewed on a memory oscilloscope (Tektronix, Courtaboeuf, France). Mediumsized spiny neurons of the Acb were clearly distinguished from fast-spiking interneurons by their basic electrophysiological properties, i.e., a low rate of spontaneous firing, spike duration and a single spike discharge in response to stimulation of cortical inputs (Wilson 1993; Mahon et al. 2001; Slaght et al. 2004; Mallet et al. 2005). During these experiments, the electrical activity of Acb cells was examined on-line and stored with a Digital Tape Recorder (DTR-1404, Biologic, Claix, France).

\section{Labeling of the Acb neurons}

Recorded neurons were labeled using juxtacellular injection of neurobiotin $(\mathrm{Nb})$ (Pinault 1996). Briefly, positive current pulses (1-8 nA, $200 \mathrm{~ms}$ duration) were applied at a frequency of $2.5 \mathrm{~Hz}$ through the bridge circuit of the amplifier. The current was slowly increased, and the electrode was advanced by steps of $1 \mu \mathrm{m}$ (LSS-1000 Inchworm Motor Positioning System, Burleigh Instruments, Fishers, NY, USA) until the cell discharge was driven by the injected current. Current pulses were applied for a 10 30 min period to obtain a reliable labeling of neuronal processes.

Two to five hours after the last injection, the animal received a lethal dose of pentobarbital $(3 \mathrm{ml} / \mathrm{kg}$, i.p.; Sanofi, Libourne, France) and Heparin ( $1 \mathrm{ml} / \mathrm{kg}$, i.p.; Choay, Paris, France) before being perfused transcardially with $200 \mathrm{ml}$ of $10 \%$ Ringer solution followed by $500 \mathrm{ml}$ of $0.2 \%$ glutaraldehyde (Prolabo, Fontenay s/bois, France) and $4 \%$ paraformaldehyde (CARLO ERBA, Val de Reuil, France) in $0.1 \mathrm{M} \quad \mathrm{NaH}_{2} \mathrm{PO}_{4} \mathrm{H}_{2} \mathrm{O} / \mathrm{Na}_{2} \mathrm{HPO}_{4}$ (phosphate buffer, pH 7.4). In all experiments, brains were post-fixed overnight at $4{ }^{\circ} \mathrm{C}$ in the same fixative solution with or without glutaraldehyde and then immersed in $30 \%$ sucrose at $4{ }^{\circ} \mathrm{C}$ until sectioned. Fifty-micrometer-thick sections were cut in the horizontal plane on a sliding freezing microtome. Sections were collected sequentially in 12 receptacles containing phosphate buffer for direct processing.

\section{Visualization of the Acb neurons}

In order to visualize the relationships of labeled dendrites and axon collaterals with the compartmental structure of the Acb (i.e., the shell and core subregions, including their smaller compartments) sections were double-stained for $\mathrm{Nb}$ and $\mathrm{CaB}$. In the procedures described below, all intermediate steps between different incubations included three rinses of the specifically indicated buffers for 10 min each. First, the sections were rinsed in phosphate buffer supplemented with $0.15 \mathrm{M} \mathrm{NaCl}$ (phosphate buffered saline [PBS], pH 7.4), and subsequently incubated in ABC-kit ([1:1] mixture of reagents $\mathrm{A}$ and $\mathrm{B}$; Vector, Burlingame, CA, USA) in PBS and $0.5 \%$ Triton X-100 (PBS-Tx; Merck) overnight at $4^{\circ} \mathrm{C}$. Thereafter, a rinse with phosphate buffer was followed by staining with Cobalt enhanced DAB-Ni substrate: $0.025 \mathrm{~g}$ DAB, $1 \mathrm{ml} 1 \%$ nickel-ammonium sulfate, $1.25 \mathrm{ml} 1 \%$ Cobalt chloride, $20 \mu \mathrm{l}$ of $30 \% \mathrm{H}_{2} \mathrm{O}_{2}$ in $50 \mathrm{ml} \mathrm{PB}$, for $10-30 \mathrm{~min}$ at rt. The progress of staining was frequently monitored with the aid of a light microscope. As soon as nonspecific background staining became visible, the reaction was terminated by several rinses in phosphate buffer, followed by rinsing with TBS-Tx and incubation in mouse anti-CaB (Sigma; 1:2,000) overnight at $4^{\circ} \mathrm{C}$. After TBS-Tx rinse, the sections were incubated with goat anti-mouse serum (1:50; Dako, Denmark) for $60 \mathrm{~min}$ at rt. Following rinsing with TBS-Tx, the sections were incubated with mPAP (1:100; Dako, Denmark) for $60 \mathrm{~min}$ at rt. Next, a Tris- $\mathrm{HCl}(\mathrm{pH}$ 7.6) rinse was followed by staining with DAB $(1 \mathrm{ml}$ DAB $[5 \mathrm{mg} /$ $10 \mathrm{ml}$ ], $9 \mathrm{ml}$ Tris- $\mathrm{HCl}$, pH 7.6 and $3.3 \mu 130 \% \mathrm{H}_{2} \mathrm{O}_{2}$ ) for $15 \mathrm{~min}$ at rt. After final rinses with Tris- $\mathrm{HCl}(\mathrm{pH}$ 7.6), the sections were mounted on glass slides from a Tris- $\mathrm{HCl}(\mathrm{pH}$ 7.6) solution, containing $0.2 \%$ gelatin, and air-dried. The mounted sections were dehydrated and coverslipped as described above.

\section{Anatomical analysis}

Neuronal reconstruction Labeled neurons, the boundaries of the Acb shell and core, and in a number of cases a patch compartment were traced and reconstructed from adjacent serial sections. Two-dimensional (2D) reconstructions were made from drawings performed under $10-40 \times$ objectives using a drawing tube attached to a light microscope. In total 35 neurons were reconstructed in 2D. The dendrites of each neuron could be traced from the cell body through 
adjacent sections. After all the dendritic segments had been drawn, each drawing was adjusted in such a way that it was aligned correctly with its neighbors, and the cut ends of the dendrites drawn were connected to make the reconstruction complete. Similar procedures were used to reconstruct the entire axonal field. A 3D computerized image-analysis system (Neurolucida, MicroBrightField, Inc.) was used to reconstruct the axonal and/or dendritic trajectory of a selection of neurons (11 out of 35 labeled neurons) that correspond to the different geometries found within the Acb shell and core subregions. To achieve these 3D reconstructions, cell bodies, axonal and/or dendritic arborizations, boundaries of the Acb shell/core and, in relevant cases, a patch compartment were precisely drawn under $25-63 \times$ oil immersion objectives. Furthermore, 3D models of these neurons were visualized using the Lightwave software (Newtek Inc., San Antonio, TX, USA). For this purpose a Perl script was developed. This script reads a Neurolucida data file and converts it into a Lightwave script, in which the $X, Y$, and $Z$ polygon coordinates corresponding to each structure (i.e., cell body, dendrites, axons, Acb shell/core and patch compartment boundaries) are described as 3D objects. The shrinkage that occurs in the $z$-axis of the brain sections during dehydration was corrected with the software. Models were then processed for solid surface rendering using the Lightwave software. The 3D-reconstructed models could be rotated. In addition, light sources and camera could be adjusted to enhance the $3 \mathrm{D}$ appearance of the reconstructed neurons when represented in $2 \mathrm{D}$ pictures.

Polar histograms The orientation of the dendritic trees of the reconstructed neurons were analyzed by generating polar histograms of their somatodendritic morphology by calculating the maximum length of dendrite and axon in each $15^{\circ}$-wide sectors around the cell body in each plane of the section from Neurolucida data files in Neuroexplorer (MicroBrightField, Inc.).

Geometrical parameters of labeled neurons in the Acb The orientation of the dendritic field of each labeled neuron $(n=35)$ was determined using the 2D reconstructions. First, the dimension of the dendritic arborization along the dorsoventral axis was measured on the basis of the number of horizontal sections in which dendrites of the filled neuron were present multiplied by the thickness of the sections, taking into account the shrinkage factor. Second, the mediolateral and rostrocaudal extensions of the dendritic field were determined in the horizontal reconstructions by measuring the distance between the tips of the farthest medially and laterally or rostrally and caudally extending
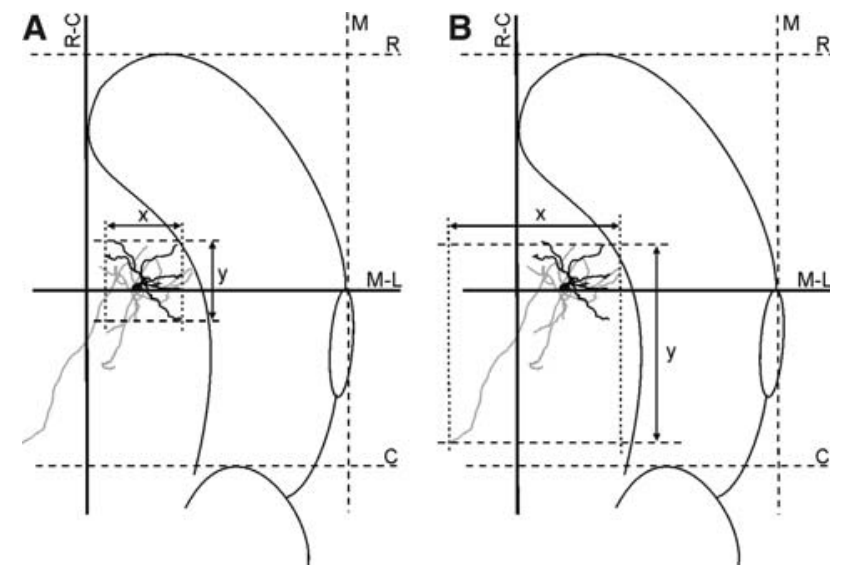

Fig. 1 Horizontal sections of the nucleus accumbens (Acb) illustrating the axes of reference used to measure the extent of the dendritic and axonal arborizations. a The mediolateral $(x)$ and rostrocaudal $(y)$ extent of the dendritic field was measured, relative to the M-L and R-C axis. The $\mathrm{M}-\mathrm{L}$ axis passes along the most rostral extension of the major island of Calleja. The R-C axis passes along the medial aspect of the curved anterior limb of the anterior commissure rostrally and its lateral aspect caudally. b The mediolateral $(x)$ and rostrocaudal $(y)$ extent of the axonal field was measured, relative to the M-L and R-C axis

dendrites (black; Fig. 1a). A similar procedure was followed to measure the distribution of axon collaterals of the labeled neurons (gray; Fig. 1b). Third, we analyzed the maximum length of dendrites around the cell body in each plane of the section to determine any orientation (or polarization) of the dendritic field.

Statistical analysis The group of neurons $(n=19)$ in which the entire neuron could be visualized, including the cell body, dendritic arborizations and local axon collaterals, was subdivided into three categories related to the degree to which dendritic arborizations and local axonal collaterals overlap with each other. The three categories include: (1) a group of MSN with 'full overlap' of dendrites and local axon collaterals, (2) a group with 'partial overlap', and (3) a group with 'no overlap'. To test whether the extent to which the axonal field expands outside the dendritic field of MSN was significantly different between the Acb shell and the core, we used the two-tailed nonparametric Mann-Whitney test.

Iontophoretic BDA injections

The above-described juxtacellular injection experiments were supplemented by 'classical' tracer experiments with small injections of the tracer biotinylated dextran amine (BDA), as performed in the context of an earlier published study (Van Dongen et al. 2005). Animal surgery, anterograde tracer injections, perfusion, fixation, storage of the 
brain, sectioning of the brain, anterograde tracer histochemistry and double staining procedure for the tracer and calbindin- $\mathrm{D}_{28 \mathrm{kDa}}$ were performed as described previously (Van Dongen et al. 2005).

\section{Data analysis}

Neuronal reconstructions were performed as described earlier. In short, labeled neurons, the boundaries of the Acb shell and core, as well as nearby located patch compartments, were traced and reconstructed from adjacent serial sections. Two-dimensional (2D) reconstructions were made from drawings performed under 10-40 $\times$ objectives using a drawing tube attached to a light microscope and transferred to CorelDraw 9.0 to construct the final chartings.

Final preparation of all half-tone figures, including contrast enhancement, was done using Adobe Photoshop 5.5.

\section{Results}

Characterization of the subregional and compartmental structure of the Acb

To differentiate between the Acb shell and core subregions, as well as between the compartments therein, we employed the immunoreactivity patterns of the calcium-binding protein calbindin- $\mathrm{D}_{28} \mathrm{kDa}(\mathrm{CaB})$ as an established maker (Fig. 2). In accordance with numerous previous studies (e.g., Zahm and Brog 1992; Jongen-Rêlo et al. 1993, 1994), the Acb core subregion shows strong immunoreactivity for $\mathrm{CaB}$ while the Acb shell is only very weakly immunoreactive. Within the Acb core, smaller compartments of light immunoreactivity, i.e., the patches, stand out against the generally strongly stained matrix. Although it has been established that the weaker immunoreactive patches in the rostral and caudal core might have different neurochemical and connectional characteristics (Voorn et al. 1989; Groenewegen et al. 1996), for the purpose of the present study we consider all lightly stained compartments in the Acb core as belonging to the patch compartment. In view of the fact that the injected cells are relatively small and therefore present in only a few (three to six) adjacent sections of the Acb, we decided to stain only for $\mathrm{CaB}$ and not for other potential markers of compartments. As a result, it was not possible to differentiate between smaller compartments within the Acb shell subregion.

General features of the injected neurons

Thirty-five injected neurons were analyzed, 21 in the Acb shell and 14 in the Acb core (Table 1; Fig. 2). In slightly more than half of these cases $(n=19)$ the entire neuron could be visualized, including the cell body, dendritic arborizations and local axon collaterals, whereas the remaining neurons $(n=16)$ showed sufficient filling of only the cell bodies and dendrites. All 35 injected neurons were identified as MSN by their morphological features. From the cell bodies of these MSN 3-6 primary dendrites radiated and strongly branched within the Acb. In the shell of the Acb, the dimensions of the dendritic tree of MSN ranged from 62 up to $375 \mu \mathrm{m}$ rostrocaudally, $150-437 \mu \mathrm{m}$ mediolaterally, and $150-400 \mu \mathrm{m}$ dorsoventrally (Table 1 ). In the core, these figures were $100-312 \mu \mathrm{m}$ in the rostrocaudal dimension, $125-325 \mu \mathrm{m}$ mediolaterally and 150-350 $\mu \mathrm{m}$ dorsoventrally (Table 1). All axons originated from the cell body forming a main stem from which several thinner collaterals with many varicosities and terminal boutons arose. The dimensions of the extending recurrent axon collaterals of MSN ranged from 275 up to $575 \mu \mathrm{m}$ rostrocaudally, 200-450 $\mu \mathrm{m}$ mediolaterally, and 200 $500 \mu \mathrm{m}$ dorsoventrally in the Acb shell. For MSN in the core these figures were $225-725 \mu \mathrm{m}$ in the rostrocaudal direction, 125-675 $\mu \mathrm{m}$ mediolaterally, and 150-400 $\mu \mathrm{m}$ dorsoventrally (Table 1). To analyze a possible difference of the extent to which the axonal field expands outside the dendritic field of MSN between the Acb shell and the core, we used the two-tailed nonparametric Mann-Whitney test, but we found no significant difference.

Nucleus accumbens core: location and geometrical aspects of injected neurons

\section{Location}

Fourteen MSN were injected in the core. These MSN were located in the rostromedial part of the nucleus (Fig. 2). Twelve MSN were located in the matrix compartment, while two MSN were located in a patch compartment. Among the MSN located in the matrix, two MSN were lying close to a patch compartment (neurons 4L-III and 8), and four MSN were situated along the shell-core boundary (neurons 9-I, 10R-I, 10L and 5-IV). One neuron was located close to the anterior commissure (neuron 1), while the remaining MSN were distributed across the matrix of the medial core (Fig. 2).

Geometrical aspects of injected neurons The analyses of the 3D aspects of the injected neurons showed that the dimensions of the dendritic arborizations were not equal in all three dimensions. In other words, the geometry of the dendrites of all individual neurons showed a flattened shape and, therefore, showed a preferred orientation (Fig. 3). Considering the orientation in the coronal plane, 
Fig. 2 Overview of the location and dendritic orientation of neurobiotin-injected neurons in the nucleus accumbens (Acb) represented in eight equally spaced horizontal sections that are 'counterstained' for the calcium-binding protein calbindin- $\mathrm{D}_{28 \mathrm{kDa}}$ to reveal the Acb shell and core subregions. The sections are arranged from dorsal (a) to ventral (h). Borders of Acb shell and core subregions are indicated with dashed lines. The transverse and sagittal planes dividing the Acb shell and core subregions into different subareas are also indicated (for a description of the criteria to position these planes, see Van Dongen et al. 2005). Each dot represents a single MSN. The blue dots indicate MSN with a rostralmedial to caudal-lateral orientation (schematically indicated with the line through the dot). The red dots represent MSN with a rostral-lateral to caudal-medial orientation (again schematically indicated with the line through the dot). The black dots encompass MSN that cannot be categorized into either the blue or red group.

Abbreviations: $a c$ anterior commissure, $A c b$ nucleus accumbens, $C B$ striatal cell bridges, ICjM major island of Calleja, $L S$ lateral shell, $L V$ lateral ventricle, $O T$ olfactory tubercle, $v C P u$ ventral caudateputamen. Scale bar in $\mathrm{H}=500 \mu \mathrm{m}$ and applies to all levels

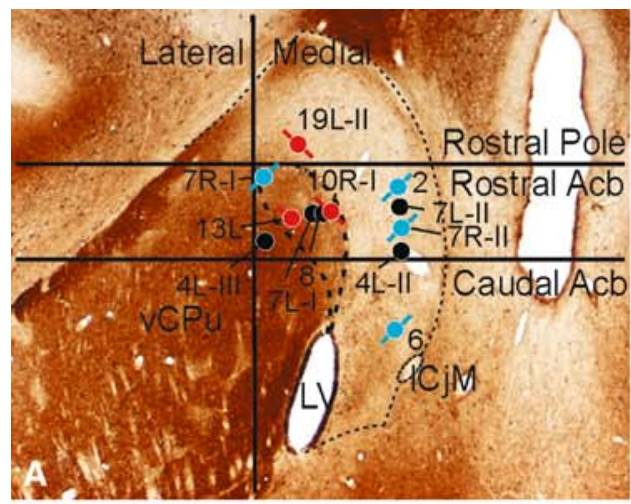

Bregma $-6.10 \mathrm{~mm}$

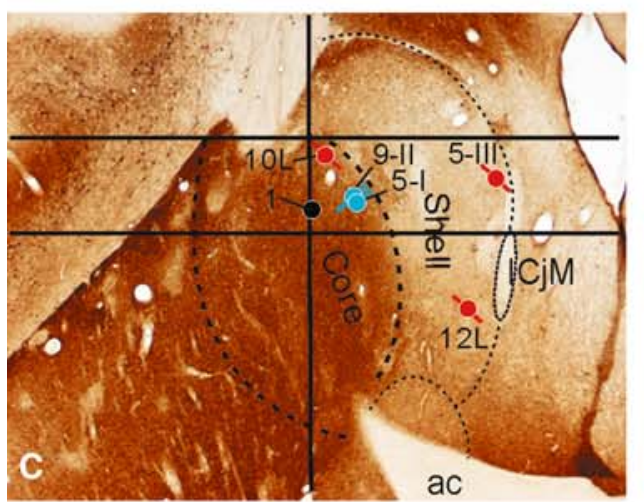

Bregma $-6.82 \mathrm{~mm}$

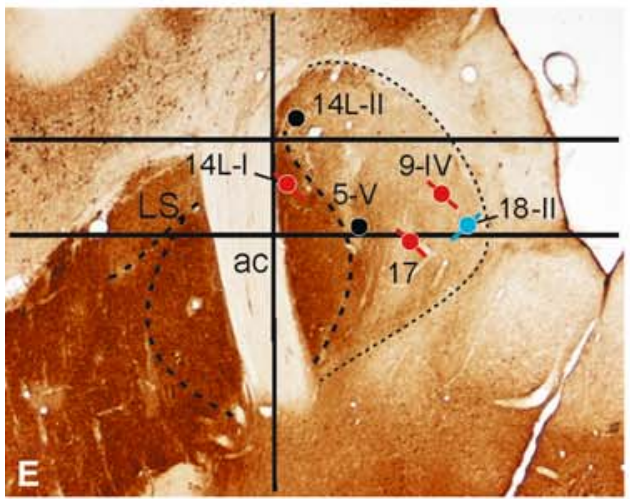

Bregma $-7.34 \mathrm{~mm}$

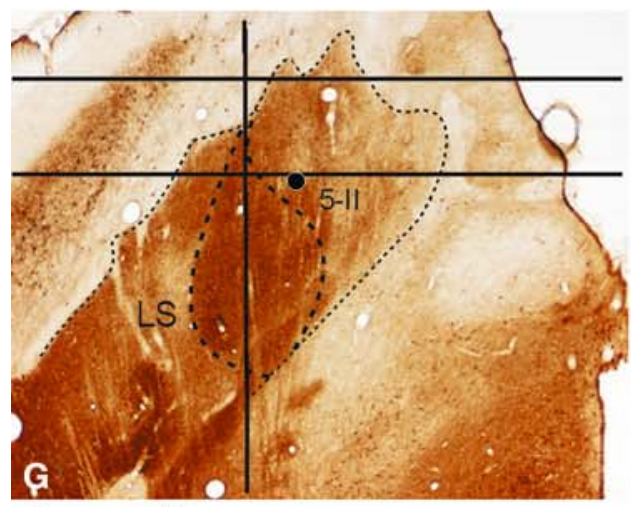

Bregma $-7.80 \mathrm{~mm}$

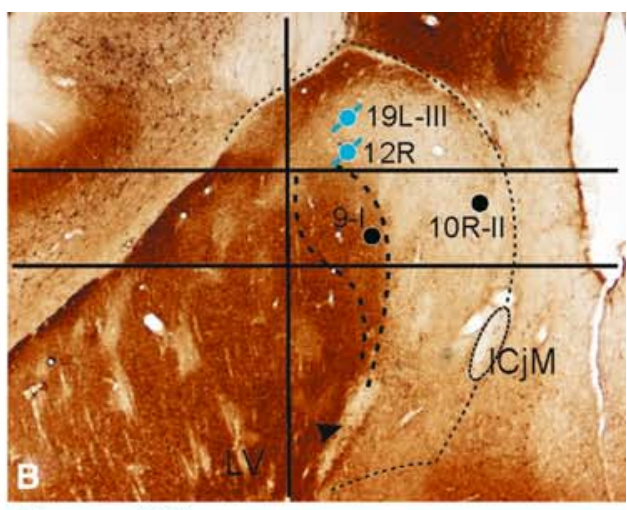

Bregma $-6.60 \mathrm{~mm}$

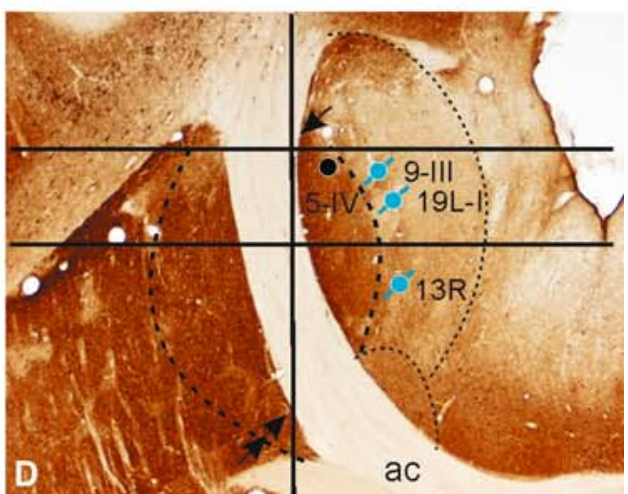

Bregma $-7.10 \mathrm{~mm}$

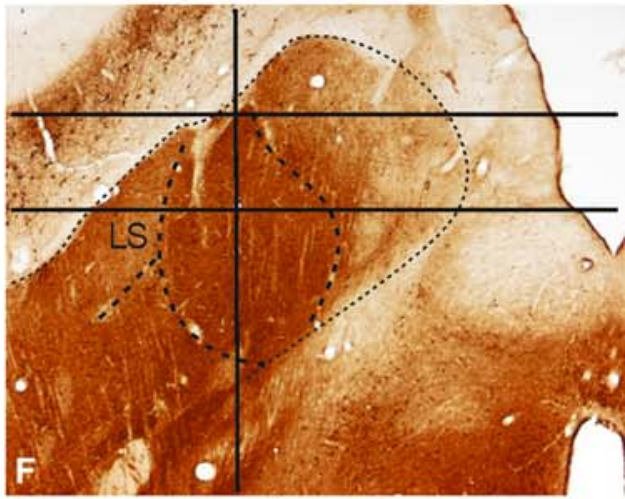

Bregma $-7.60 \mathrm{~mm}$

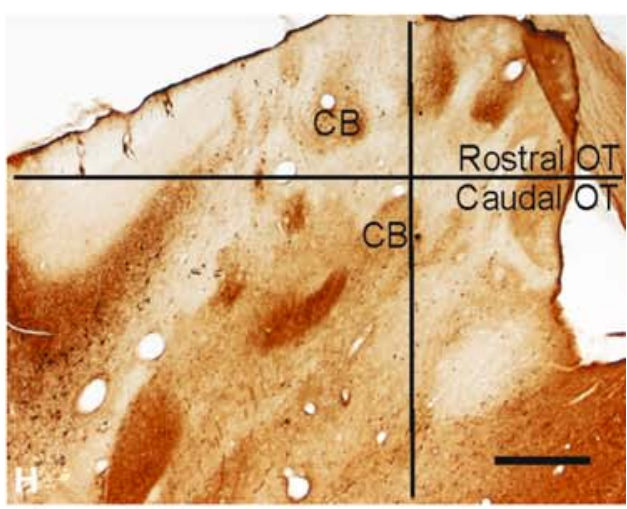

Bregma $-8.60 \mathrm{~mm}$ 
Table 1 Distance from the shell-core boundary, extension of the dendritic and axonal fields, extent to which the axonal field expands outside the dendritic field, and orientation of the dendritic field of medium-sized spiny projection neurons in the rat nucleus accumbens shell and core

\begin{tabular}{|c|c|c|c|c|c|c|c|c|c|c|c|c|}
\hline \multirow[t]{2}{*}{ Neuron } & \multirow{2}{*}{$\begin{array}{l}\text { Dist. from } \\
\text { shell-core } \\
\text { border }\end{array}$} & \multicolumn{3}{|c|}{$\begin{array}{l}\text { Extension of dendritic } \\
\text { field (in } \mu \mathrm{m} \text { ) }\end{array}$} & \multicolumn{3}{|c|}{$\begin{array}{l}\text { Extension of axonal } \\
\text { field (in } \mu \mathrm{m})\end{array}$} & \multicolumn{3}{|c|}{$\begin{array}{l}\text { Axonal field expand } \\
\text { dendritic field (in } \mu \mathrm{m} \text { ) }\end{array}$} & \multicolumn{2}{|c|}{$\begin{array}{l}\text { Orientation } \\
\text { of dendritic field }\end{array}$} \\
\hline & & $\mathrm{D}-\mathrm{V}$ & $\mathrm{R}-\mathrm{C}$ & M-L & $\mathrm{D}-\mathrm{V}$ & $\mathrm{R}-\mathrm{C}$ & M-L & D-V & $\mathrm{R}-\mathrm{C}$ & M-L & RC-ML & DV-ML \\
\hline \multicolumn{13}{|l|}{ Shell } \\
\hline 10R-II & 375 & 250 & 275 & 200 & 350 & 525 & 400 & 100 & 250 & 200 & $\mathrm{v}$ & DM-VL \\
\hline $12 \mathrm{R}$ & 62 & 300 & 200 & 250 & 400 & 350 & 375 & 100 & 150 & 125 & RM-CL & DM-VL \\
\hline 5 -II & 125 & 200 & 300 & 350 & 250 & 450 & 325 & 50 & 150 & - & $\mathrm{v}$ & DM-VL \\
\hline 14L-II & 125 & 200 & 375 & 150 & 200 & 475 & 300 & - & 100 & 150 & $\mathrm{v}$ & $\mathrm{v}$ \\
\hline 19L-I & 125 & 250 & 250 & 250 & 300 & 450 & 450 & 50 & 200 & 150 & RM-CL & DL-VM \\
\hline 19L-II & 125 & 200 & 225 & 275 & 200 & 375 & 325 & - & 150 & 50 & RL-CM & DM-VL \\
\hline 19L-III & 187 & 250 & 225 & 150 & 250 & 375 & 425 & - & 150 & 275 & RM-CL & DM-VL \\
\hline 2 & 250 & 350 & 300 & 350 & 400 & 400 & 375 & 50 & 100 & 25 & RM-CL & DM-VL \\
\hline 4L-II & 250 & 250 & 200 & 225 & 250 & 275 & 200 & - & 75 & - & $\mathrm{v}$ & DM-VL \\
\hline $7 \mathrm{R}-\mathrm{II}^{\mathrm{a}}$ & 250 & 400 & 325 & 325 & 500 & 575 & 450 & 100 & 250 & 125 & RM-CL & DL-VM \\
\hline 5-III & 500 & 300 & 187 & 187 & - & - & - & - & - & - & RL-CM & DL-VM \\
\hline 9-III & 125 & 250 & 62 & 175 & - & - & - & - & - & - & RM-CL & $\mathrm{v}$ \\
\hline $13 R$ & 125 & 200 & 187 & 187 & - & - & - & - & - & - & RM-CL & DM-VL \\
\hline 17 & 187 & 200 & 250 & 187 & - & - & - & - & - & - & RL-CM & DM-VL \\
\hline $5-\mathrm{V}$ & 62 & 250 & 250 & 125 & - & - & - & - & - & - & $\mathrm{v}$ & $\mathrm{v}$ \\
\hline 6 & 250 & 250 & 187 & 187 & - & - & - & - & - & - & RM-CL & DM-VL \\
\hline 7L-II & 250 & 250 & 187 & 187 & - & - & - & - & - & - & $\mathrm{v}$ & $\mathrm{v}$ \\
\hline 9-IV & 375 & 200 & 250 & 250 & - & - & - & - & - & - & RL-CM & $\mathrm{v}$ \\
\hline $12 \mathrm{~L}$ & 250 & 200 & 187 & 250 & - & - & - & - & - & - & RL-CM & VL \\
\hline 18 & 500 & 150 & 250 & 437 & - & - & - & - & - & - & RM-CL & DM-VL \\
\hline \multicolumn{13}{|l|}{ Core } \\
\hline 7R-I & 187 & 200 & 125 & 275 & 250 & 300 & 225 & 50 & 175 & 100 & RM-CL & $\mathrm{v}$ \\
\hline $7 \mathrm{~L}-\mathrm{I}^{\mathrm{a}}$ & 187 & 150 & 250 & 200 & 550 & 300 & 200 & 550 & 300 & 200 & $\mathrm{v}$ & DL \\
\hline $14 \mathrm{~L}-\mathrm{I}$ & 125 & 300 & 275 & 225 & 350 & 225 & 125 & 50 & - & - & RL-CM & DM-VL \\
\hline $5-\mathrm{I}$ & 125 & 150 & 100 & 125 & 150 & 725 & 675 & 150 & 725 & 675 & $\mathrm{RM}$ & $\mathrm{v}$ \\
\hline 1 & 375 & 250 & 300 & 325 & 250 & 300 & 300 & - & - & - & $\mathrm{v}$ & $\mathrm{v}$ \\
\hline 9-I & 62 & 200 & 200 & 275 & 350 & 375 & 300 & 150 & 175 & 25 & $\mathrm{v}$ & $\mathrm{v}$ \\
\hline $10 \mathrm{R}-\mathrm{I}$ & 62 & 350 & 250 & 275 & 400 & 425 & 400 & 50 & 175 & 125 & RL-CM & DM-VL \\
\hline 5 -IV & 62 & 250 & 312 & 187 & - & - & - & - & - & - & $\mathrm{v}$ & VL \\
\hline 8 & 125 & 150 & 250 & 125 & - & - & - & - & - & - & $\mathrm{v}$ & DL \\
\hline $10 \mathrm{~L}$ & 62 & 250 & 250 & 125 & - & - & - & - & - & - & RL-CM & DL-VM \\
\hline 9-II & 125 & 250 & 312 & 187 & - & - & - & - & - & - & RM-CL & DM-VL \\
\hline 4L-III & 375 & 250 & 250 & 125 & - & - & - & - & - & - & $\mathrm{v}$ & $\mathrm{v}$ \\
\hline $13 \mathrm{~L}$ & 250 & 200 & 250 & 125 & - & - & - & - & - & - & RL-CM & DM-VL \\
\hline
\end{tabular}

a $n=2$

$v=$ variety of dendritic orientations

it appeared that five MSN showed a dendritic orientation in dorsal-medial to ventral-lateral direction, while four MSN showed a preference for the dorsal-lateral to ventral-medial direction. The remaining five MSN showed a variety of dendritic orientations. An example of an MSN located in the matrix compartment with a dorsal-medial to ventral-lateral dendritic orientation is illustrated in Fig. 4.
The specific geometry of the dendritic arborizations of MSN appears to be profoundly influenced by the proximity of the shell-core boundary, a patch compartment or the anterior commissure, as illustrated in Figs. 5, 6, 7, 8, 9 and 10. An example of an MSN close to the shell-core boundary is illustrated in Fig. 5. The geometry of the dendritic arborizations of this neuron (neuron 5-IV) is strongly asymmetric, such that the dendrites are mainly 


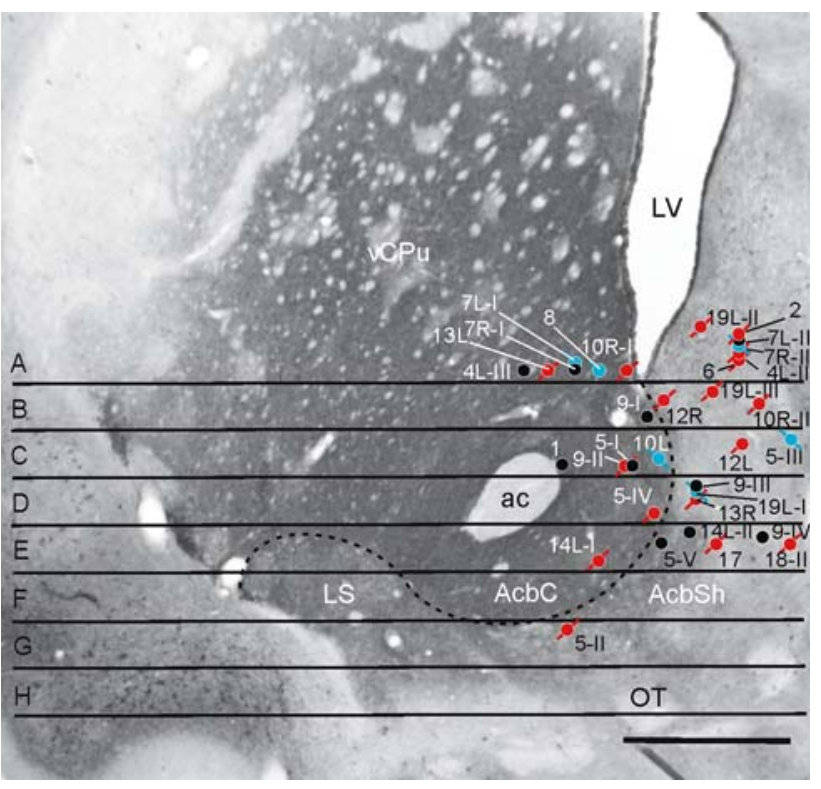

Fig. 3 Overview of the dendritic orientation of neurobiotin-injected neurons in the Acb represented in a coronal section that is 'counterstained' for the calcium-binding protein calbindin- $\mathrm{D}_{28} \mathrm{kDa}$ to reveal the Acb shell and core subregions. The border between the Acb shell and core subregions is indicated with a dashed line. The horizontal planes that correspond to eight equally spaced horizontal sections (see Fig. 2) are also indicated. Each dot represents a single MSN with a particular dendritic orientation. The blue dots stand for MSN with a dorsal-lateral to ventral-medial orientation (schematically indicated with the line through the dot). The red dots represent MSN with a dorsal-medial to ventral-lateral orientation (again schematically indicated with the line through the dot). The black dots encompass MSN that cannot be categorized into either the blue or red group. Abbreviations: $a c$ anterior commissure, $A c b C$ nucleus accumbens core, AcbSh nucleus accumbens shell, $L S$ lateral shell, $L V$ lateral ventricle, $O T$ olfactory tubercle, $v C P u$ ventral caudateputamen. Scale bar is $500 \mu \mathrm{m}$

oriented parallel to and away from the boundary of shell and core, without dendrites crossing this boundary. Such features could also be observed in experiments with small BDA injections that label multiple MSN in a small cluster close to the shell-core boundary (Fig. 6a, b). Likewise, dendrites of neurons lying close to a patch compartment (Fig. 7) or to the anterior commissure (not illustrated) appeared to approach, but not cross the boundaries, the main dendritic orientation diverted away from the patch or the fiber bundle. Examples of the influence exerted by the compartmental boundaries on the dendritic arborizations are illustrated with a single BDA-labeled neuron (neuron 03091) and neurons 7L-I and 4L-III (Figs. 7, 8, 9). The cell body of neuron 4L-III was situated within one of the curvatures of a patch compartment, and its dendritic field curved nicely along the border of the compartment conforming to the geometry of this compartment resulting in a strongly asymmetric orientation of the dendrites related to the cell body (Fig. 8).
Nucleus accumbens shell: location and geometrical aspects of injected neurons

\section{Location}

Twenty-one MSN were injected in the shell. The majority of MSN were located in the medial part of the nucleus, two of these MSN being located along the shell-core boundary (Fig. 2).

Geometrical aspects of injected neurons Like in the Acb core, the dimensions of the dendritic arborizations of the MSN are not equal in all three dimensions, such that the geometry of the dendrites of all individual neurons showed a flattened shape and, therefore, showed a preferred orientation (Fig. 3). Unlike the Acb core, the dendritic orientations of MSN in the Acb shell are more homogeneous. Considering the orientation in coronal plane, it appeared that $12 \mathrm{MSN}$ showed a dendritic orientation in dorsal-medial to ventral-lateral direction, while four MSN showed a preference for the dorsal-lateral to ventralmedial direction. The remaining five MSN do not fit into these two larger categories and showed different dendritic orientations. Figure 10 illustrates an individual MSN located in the medial shell showing a dorsal-medial to ventral-lateral dendritic orientation.

Like in the Acb core, the specific geometry of the dendritic arborizations of MSN was profoundly influenced by the proximity of the shell-core boundary (Figs. 6, 11). Thus, as illustrated in Fig. 11, the geometry of the dendritic arborizations of neuron $5-\mathrm{V}$ is strongly asymmetric, such that the dendrites were mainly oriented parallel to and away from the boundary of shell and core, without dendrites crossing this boundary. Such features could also be observed following small BDA injections (case 03091) that labeled a cluster of MSN in close vicinity of the shellcore border (Fig. 6c, d).

The distribution of the local axon collaterals of MSN in relation to the geometry of the dendrites

The recurrent axon collaterals of MSN in both the Acb shell and core subregions terminated within and outside the dendritic field of their parent neuron. Among the population of MSN examined, a considerable variability was observed in the extension of the axon collaterals. Most likely, such variability was not due to the limitations of the injection or staining procedure, because in all the selected cases the axon collaterals were strongly stained suggesting adequate filling. In addition, in most cases the main axon could be followed over several millimeters into the ventral pallidum and beyond (see Table 1 in Van Dongen et al. 


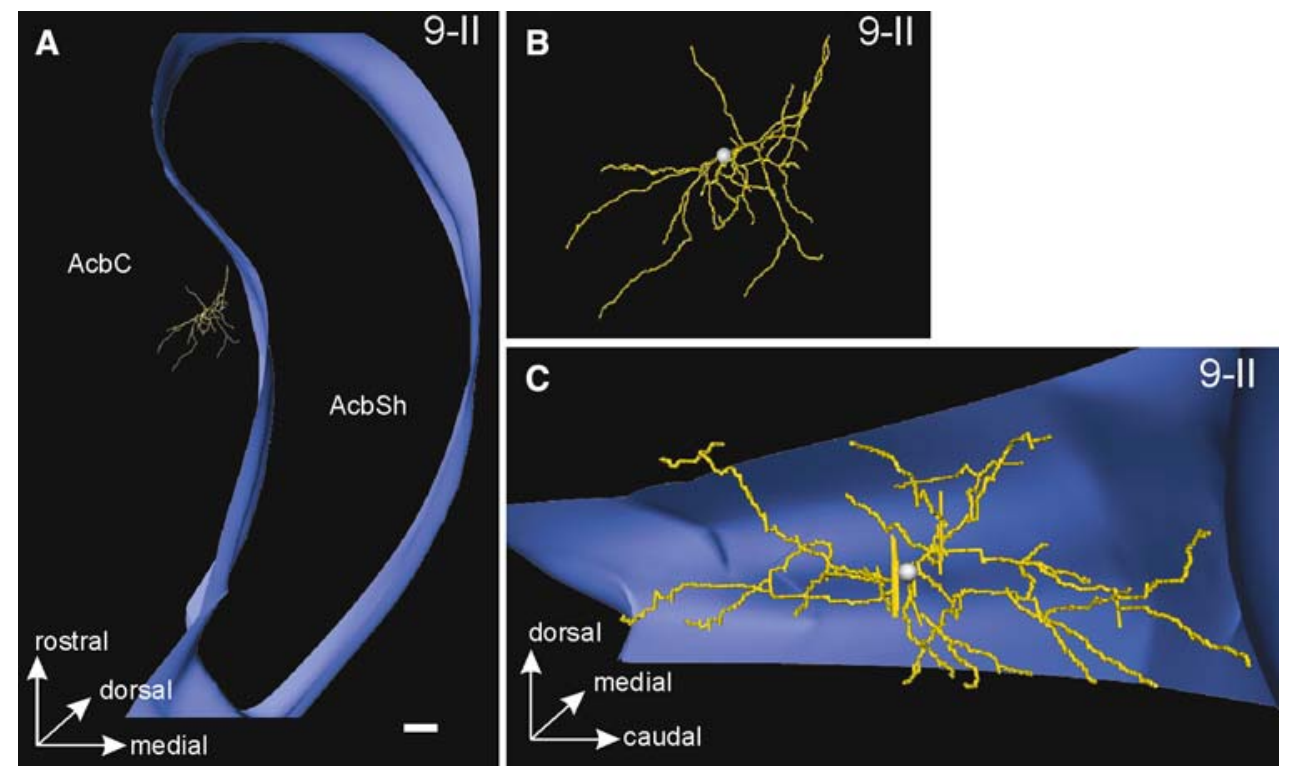

Fig. 4 Three-dimensional (3D) reconstruction of a single MSN in the Acb core subregion with a dorsal-medial-rostral to ventral-lateralcaudal dendritic orientation. a The neuron (neuron 9-II) is located in the rostromedial part of the core subregion of the Acb and examined from a dorsal view in $\mathbf{a}, \mathbf{b}$ and a lateral view in $\mathbf{c}$. In blue is indicated the contour of the Acb shell. b A zoomed view of the 3D-
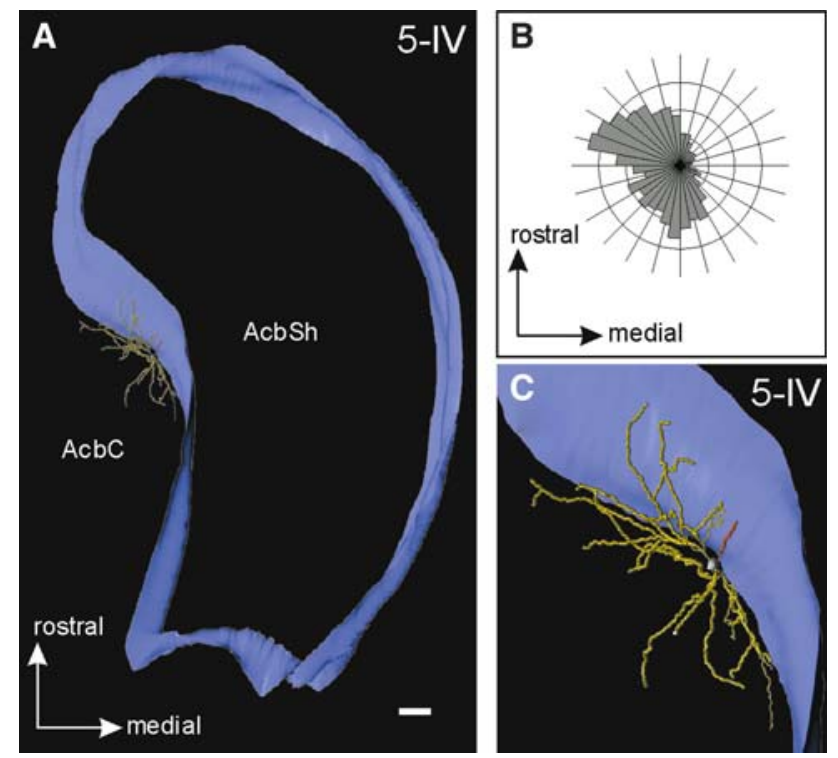

Fig. 5 Three-dimensional (3D) reconstruction of a single MSN in the Acb core subregion in close vicinity to the shell-core boundary. a The neuron (neuron 5-IV) is located in the rostromedial part of the core subregion of the Acb, along the shell-core boundary at a range of approximately $60 \mu \mathrm{m}$ from the shell-core border. In blue is indicated the contour of the Acb shell. b Polar histogram of the dendritic orientation (in gray). The polar histogram shows that the dendrites extend in rostral-lateral and caudal-lateral direction. c A zoomed view of the 3D-reconstruction of the neuron shown in a. Note that the dendritic arbors extend parallel to and away from the shell-core boundary. Abbreviations: $A c b C$ nucleus accumbens core, AcbSh nucleus accumbens shell. Scale bar is $100 \mu \mathrm{m}$ reconstruction of the neuron shown in a. c The $3 \mathrm{D}$ reconstruction shows that the dendritic arbors of the neuron are extended from dorsal-medial-rostral to ventral-lateral-caudal. Abbreviations: $A c b C$ nucleus accumbens core, AcbSh nucleus accumbens shell. Scale bar in $\mathbf{a}=100 \mu \mathrm{m}$

2005), indicating that the animal survival time was long enough to label axons over distances greater than the length of intra-accumbens collaterals. Therefore, the observed differences in the extension of the axon collaterals most likely reflected an existing structural variability in the local axonal network of Acb MSN.

\section{Nucleus accumbens core}

Eight MSN located in the medial Acb core were sufficiently labeled to allow full reconstruction of their axon. Several patterns of the distribution of the local axon collaterals in relation to the dendrites were observed. The dendrites and recurrent axon collaterals showed either full (neuron 1), partial (neurons 9-I, 7R-I, 14L-I and 10R-I) or no overlap (neurons 5-I and 7L-I). The axon collaterals of neuron 5-I showed a widely distributed network extending away from the cell body up to $1 \mathrm{~mm}$ away from the cell of origin (Fig. 12). Two MSN with a largely overlapping distribution of dendrites and local axon collaterals, but with a difference in the extension of their axon collaterals, are illustrated in Figs. 7 and 13. Neuron 7R-I shows an example of a local axonal network that extended into the adjacent subregion, i.e., the rostral pole. As can be seen in Fig. 13, three axonal branches traversed the border between the shell and core ( $\mathrm{cf}$ also Van Dongen et al. 2005). Additionally, a single BDA-injected MSN (neuron 

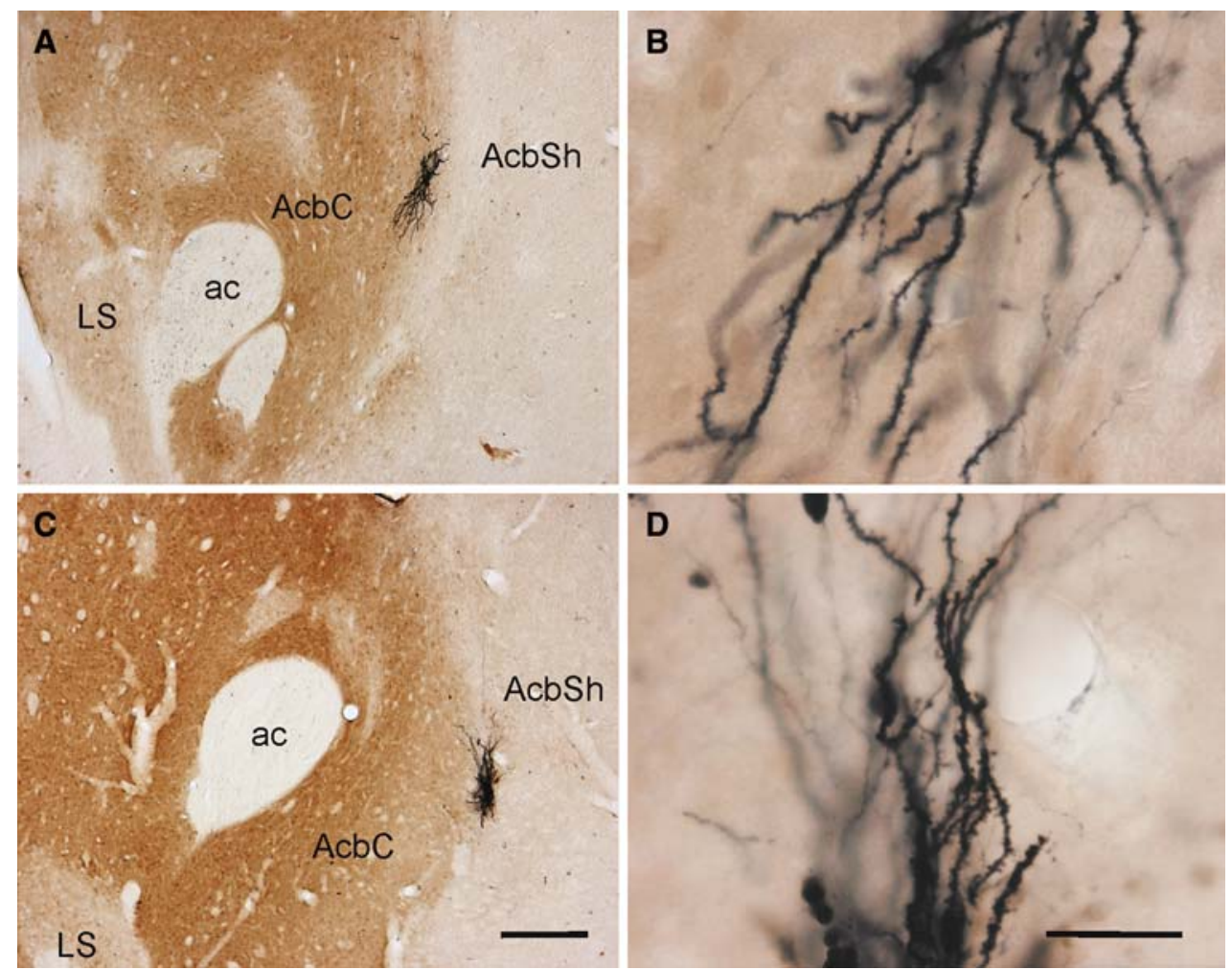

Fig. 6 Photomicrographs of two BDA injection sites in the Acb shell and core subregions in close vicinity to the shell-core boundary. a Low power photomicrograph showing the location of the BDA injection site in the Acb core. Note that the BDA-injected neurons are oriented along the shell-core border in a slightly dorsal-medial to ventral-lateral direction. b High power photomicrograph illustrating the high density of dendritic spines. Note that the BDA-injected

03091) forms an example of a local axonal network that extended to an adjacent compartment (Fig. 7). This neuron was closely related to a nearby located patch compartment (Fig. 7a, b). Interestingly, two axonal branches of this matrix neuron traversed the border with the patch to enter this compartment (Fig. 7c).

\section{Nucleus accumbens shell}

In the shell, the dendrites and recurrent axon collaterals of all 11 reconstructed MSN showed a partial overlap of dendrites and axonal collaterals. Interestingly, in most cases the axon collaterals tended to extend rostrally beyond the reach of the dendrites. A representative neuron (neuron 4L-II) is illustrated in Fig. 14.

\section{Discussion}

This study provides the first analysis of the spatial organization of both the dendritic and axonal arborizations of neurons respect the shell-core border. c Low power photomicrograph showing the location of the BDA injection site in the Acb shell. d High power photomicrograph illustrating the high density of dendritic spines. Abbreviations: $a c$ anterior commissure, AcbC nucleus accumbens core, AcbSh nucleus accumbens shell, $L S$ lateral shell. Scale bar in $\mathbf{c}=250 \mu \mathrm{m}$ and applies also to $\mathbf{a}$, scale bar in $\mathbf{d}=30 \mu \mathrm{m}$ and applies also to $\mathbf{b}$

MSN in the shell and core subregions of the rat Acb. Our principal finding is that the dendritic arborizations of MSN in both the Acb shell and core subregions are preferentially oriented, i.e., they are flattened in at least one of the 3Dplanes. Moreover, the data show that the orientations of dendritic arborizations of MSN in the Acb are influenced by the shell-core boundary, and they confirm earlier observations that the dendritic arborizations conform to compartmental (patch-matrix) boundaries (e.g., Gerfen et al. 1985; Bolam et al. 1988; Kawaguchi et al. 1989; Arts and Groenewegen 1992; Meredith et al. 1992). Thus, in the Acb shell, the dendrites of MSN situated close to the shellcore boundary are oriented parallel to or away from the border but they do not cross the border. The majority of MSN in the shell have dendritic arborizations that have a rostral-medial-dorsal to caudal-lateral-ventral orientation. In the Acb core, the dendritic arborizations of MSN likewise respect the shell-core border as well as patch-matrix boundaries. The preferred orientations of the dendrites of MSN in the core are more varied when compared to the Acb shell and the rest of the striatum. Unlike the dendrites, recurrent axon collaterals of MSN were observed to cross 

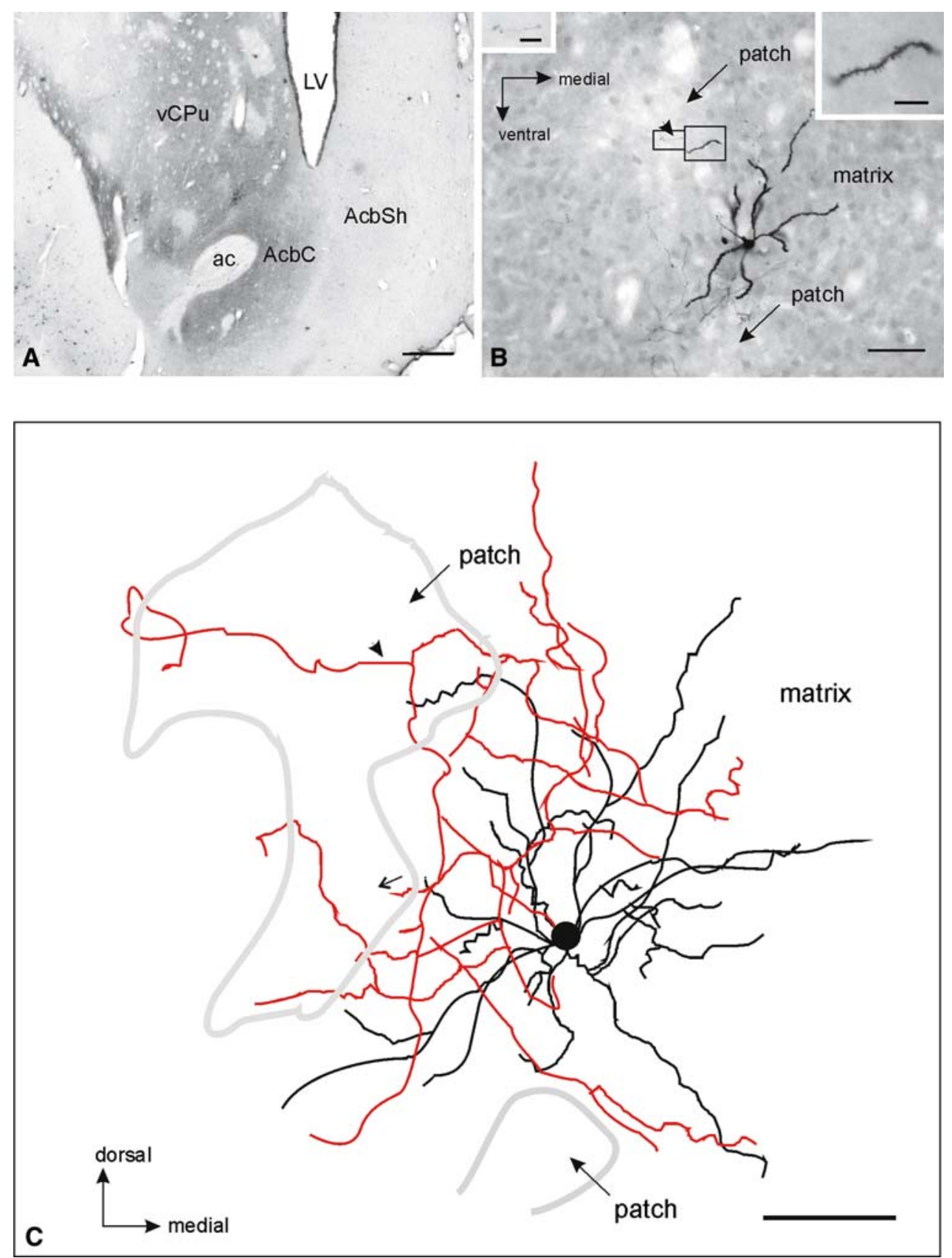

Fig. 7 Photomicrographs and two-dimensional (2D) reconstruction of both the dendrites and axons of a single BDA-labeled MSN (neuron 03091) in the matrix of the rostromedial part of the core subregion of the Acb in close vicinity to a patch compartment. a Low power photomicrograph of a coronal section through the Acb doublestained for the tracer BDA and the calcium-binding protein calbindin$\mathrm{D}_{28 \mathrm{kDa}}$ to reveal the Acb shell and core subregions and its compartments and to show the location of the neuron in the Acb core. b High power photomicrograph showing the neuron and its relationship with the adjacent patch compartments. The area enclosed by the large rectangle is shown at higher magnification in the righthand corner inset; note the high density of dendritic spines. Note also that this dendritic branch is located within the patch compartment.
The area enclosed by the small rectangle is shown at higher magnification in the left-hand corner inset; note the labeled varicose fiber. c Two-dimensional reconstruction of the neuron illustrating the orientation and distribution of its dendrites (in black) and recurrent axon collaterals (in red). The 2D reconstruction shows that the dendritic arbors of the neuron extend in dorsal-medial to ventrallateral direction. Note that the axonal network arborizes within the adjacent patch compartment (arrowhead in $\mathbf{b}, \mathbf{c}$ ). The arrow indicates the main axon leaving the Acb. Abbreviations: $a c$ anterior commissure, $A c b C$ nucleus accumbens core, AcbSh nucleus accumbens shell, $L V$ lateral ventricle, $v C P u$ ventral caudate-putamen. Scale bar in $\mathbf{a}=250 \mu \mathrm{m}$, scale bar in $\mathbf{b}=50 \mu \mathrm{m}$, scale bar in right-hand corner inset $=10 \mu \mathrm{m}$ and scale bar in left-hand corner inset $=5 \mu \mathrm{m}$ 


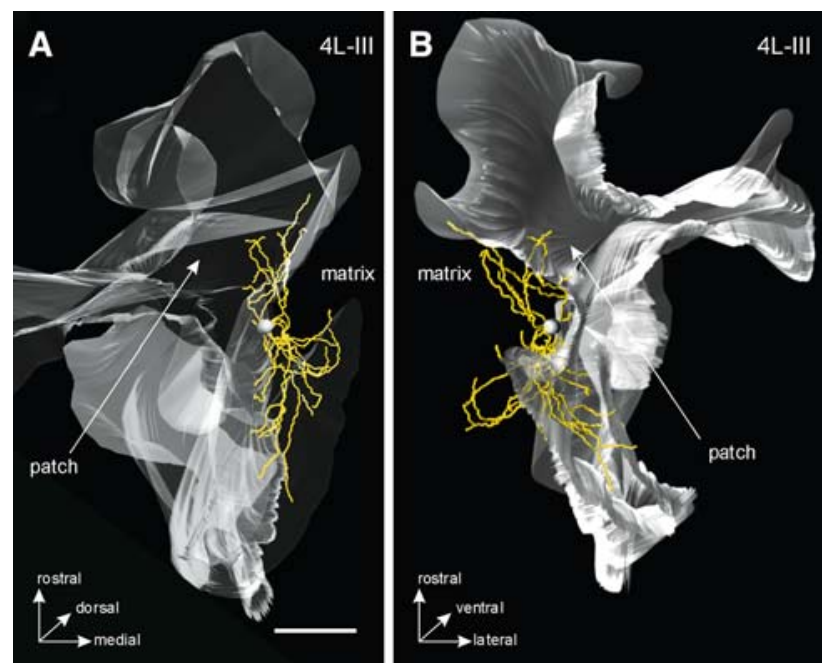

Fig. 8 Three-dimensional (3D) reconstruction of the dendritic arborizations of a single MSN in the Acb core subregion in close vicinity to a patch compartment. a, b The neuron (neuron 4L-III) is located adjacent to a patch compartment (gray) in the rostromedial part of the core subregion of the Acb. Note that its dendrites arborize along the curvatures of the patch compartment and that the dendritic arbors do not cross the border between the patch and matrix. Scale bar in $\mathbf{a}=100 \mu \mathrm{m}$

subregional (shell-core) and compartmental (patch-matrix) boundaries. Our data also show that different degrees of overlap exist between dendritic and axonal arborizations of individual MSN, including full, partial or no overlap. This suggests various ways of communication between MSN in the ventral striatum.

Meredith et al. (1992) found significant morphological differences between projection neurons in the shell and core, and patch and matrix compartments therein, on the basis of dendritic length, number of primary dendrites and spine density. Since we were primarily interested in the 3D geometry of dendritic and recurrent axonal arborizations in relationship to subregional and compartmental boundaries, we have no direct quantitative data to compare with the study of Meredith et al. (1992). However, on the basis of qualitative observations we agree with their conclusion that the spine density in the shell is lower than that in the core. The parameter we compared quantitatively between shell and core neurons, i.e., the degree of overlap between the dendritic and axonal arborizations, did not show significant differences between neurons in these two subregions of the Acb.

Spatial organization and orientation of dendritic arborizations of MSN

It has been shown previously, both in the dorsal (Gerfen et al. 1985; Penny et al. 1988; Bolam et al. 1988;

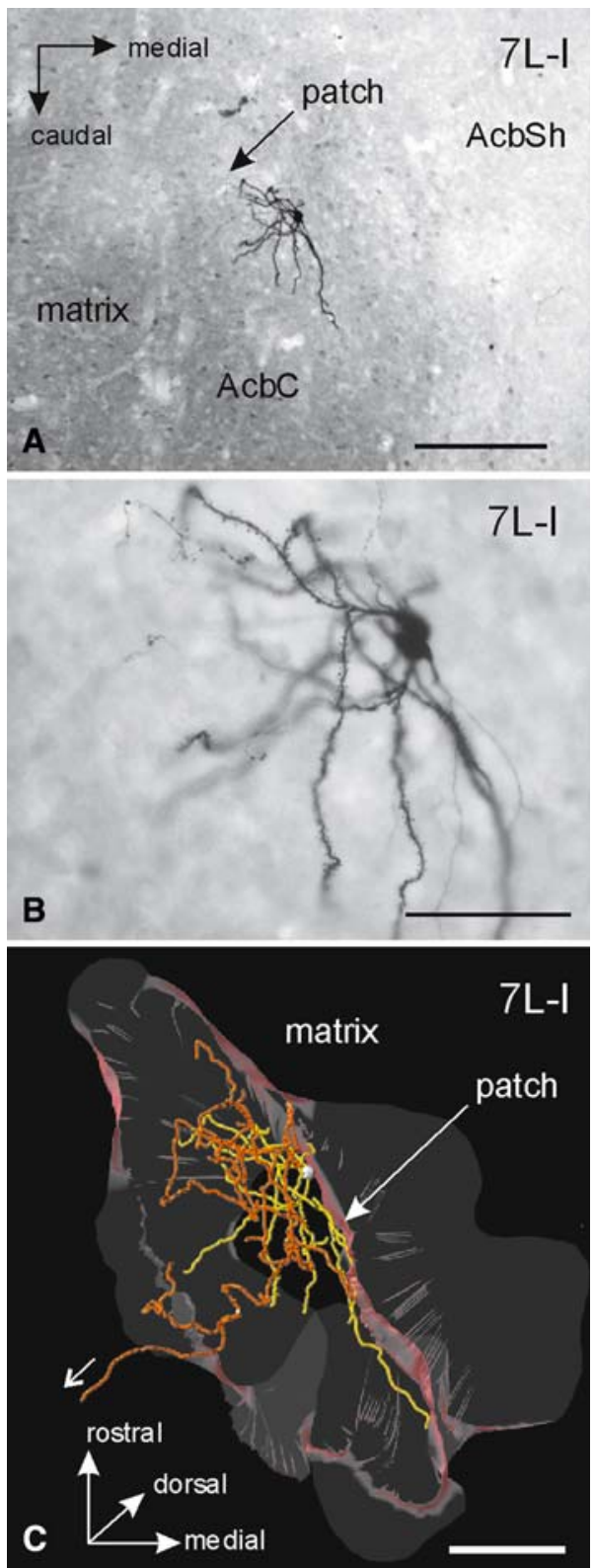

Fig. 9 Photomicrographs and 3D reconstruction of two MSN (two neurons labeled as $7 \mathrm{~L}-\mathrm{I}$ ) in a patch compartment of the rostromedial part of the core subregion of the Acb in a horizontal section through the Acb, double-stained for the tracer neurobiotin and the calciumbinding protein calbindin- $\mathrm{D}_{28} \mathrm{kDa}$ to reveal the shell and core subregions and its compartments. a Low power photomicrograph showing the location of the neurons in a patch compartment. b High power photomicrograph of the two neurons, note the high density of dendritic spines. c 3D-reconstruction of one of the neurons illustrating the distribution of its dendrites (yellow) and local axon collaterals (orange), as well as its relationship with the patch compartment. The arrow indicates the main axon leaving the Acb. Note that the dendritic arbors of the neuron extend parallel to and away from the border of the matrix compartment. Abbreviations: $A c b C$ nucleus accumbens core, AcbSh nucleus accumbens shell. Scale bar in $\mathbf{a}=150 \mu \mathrm{m}$, scale bar in $\mathbf{b}=50 \mu \mathrm{m}$ and scale bar in $\mathbf{c}=100 \mu \mathrm{m}$ 


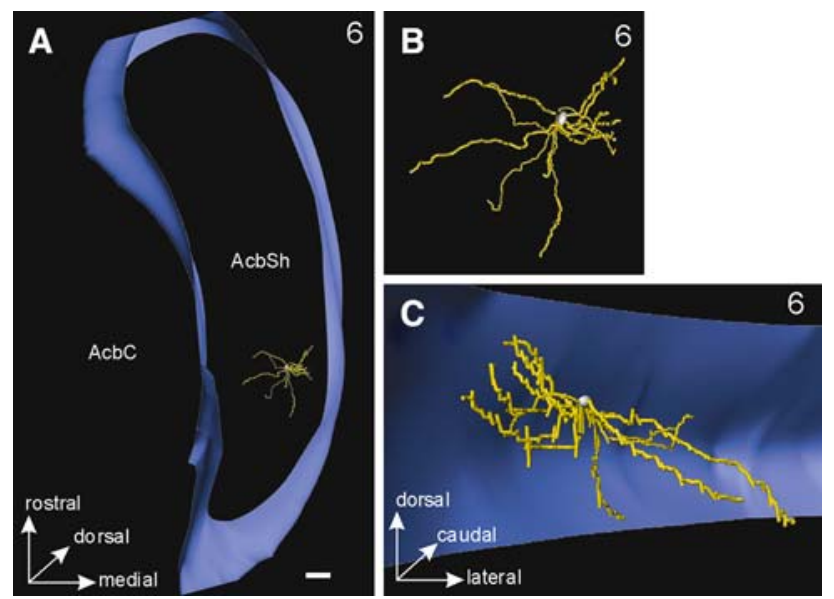

Fig. 10 Three-dimensional (3D) reconstruction of a single MSN in the Acb shell subregion with a dorsal-medial-rostral to ventral-lateralcaudal dendritic orientation. a The neuron (neuron 6) is located in the caudomedial part of the shell subregion of the Acb and examined from a dorsal view in $\mathbf{a}, \mathbf{b}$ and a rostral view in $\mathbf{c}$. In blue is indicated the contour of the Acb shell. b A zoomed view of the 3Dreconstruction of the neuron shown in a. c The 3D-reconstruction illustrates that the dendrites of the neuron are oriented from dorsalmedial-rostral to ventral-lateral-caudal. Abbreviations: $A c b C$ nucleus accumbens core, AcbSh nucleus accumbens shell. Scale bar in $\mathbf{a}=100 \mu \mathrm{m}$
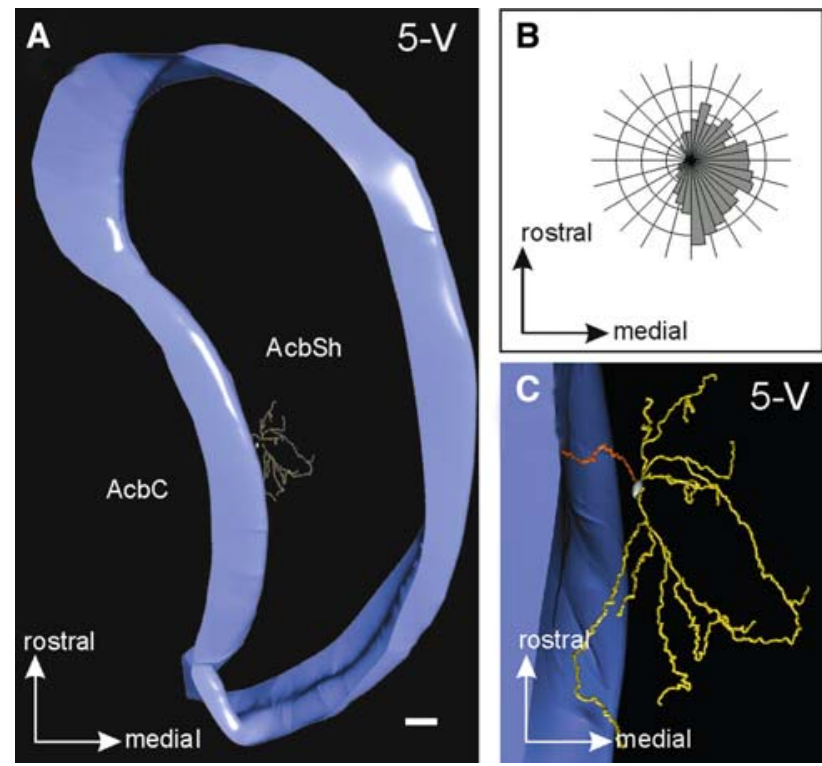

Fig. 11 Three-dimensional (3D) reconstruction of a single MSN in the Acb shell subregion in close vicinity to the shell-core boundary. a The neuron, (neuron 5-V) is located in the rostromedial part of the shell subregion of the Acb, along the shell-core boundary at a range of approximately $60 \mu \mathrm{m}$ from the shell-core border. In blue is indicated the contour of the Acb shell. b Polar histogram of the dendritic orientation (in gray). The polar histogram shows that the dendrites extend in rostral-medial and caudal-medial direction. c A zoomed view of the 3D-reconstruction of the neuron shown in $\mathbf{a}$. Note that the dendritic arbors extend parallel to and away from the shell-core boundary. Abbreviations: $A c b C$ nucleus accumbens core, AcbSh nucleus accumbens shell. Scale bar in $\mathbf{a}=100 \mu \mathrm{m}$
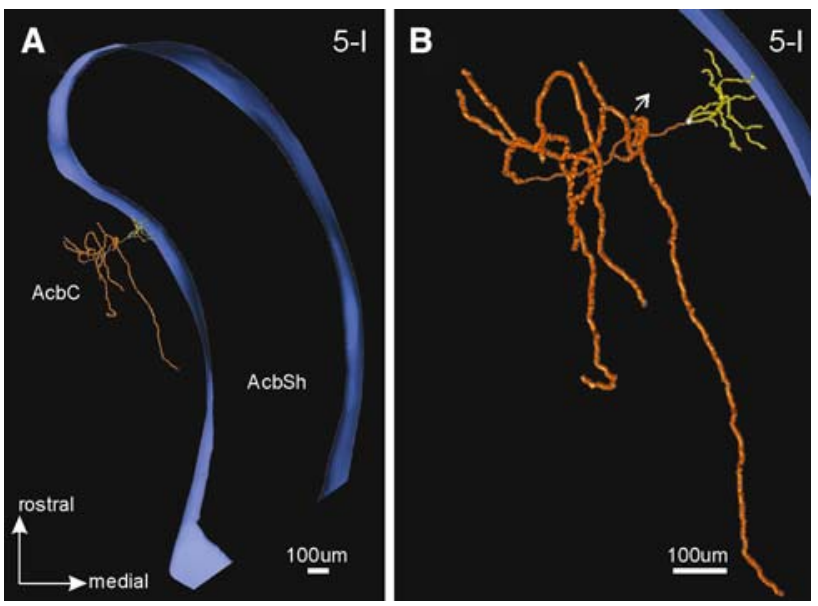

Fig. 12 Three-dimensional (3D) reconstruction of a single MSN in the Acb core subregion with a widespread distribution of local axon collaterals and lack of overlap between its dendrites and recurrent axon collaterals. a The neuron (neuron 5-I) is located in the rostromedial part of the core subregion of the Acb. In blue is indicated the contour of the Acb shell. Note the lack of overlap between dendrites and local axon collaterals and the widespread distribution of its axonal network. b A zoomed view of the 3Dreconstruction of the neuron shown in $\mathbf{a}$. The arrow indicates the main axon leaving the Acb. Abbreviations: $A c b C$ nucleus accumbens core, AcbSh nucleus accumbens shell. Scale bar is $100 \mu \mathrm{m}$
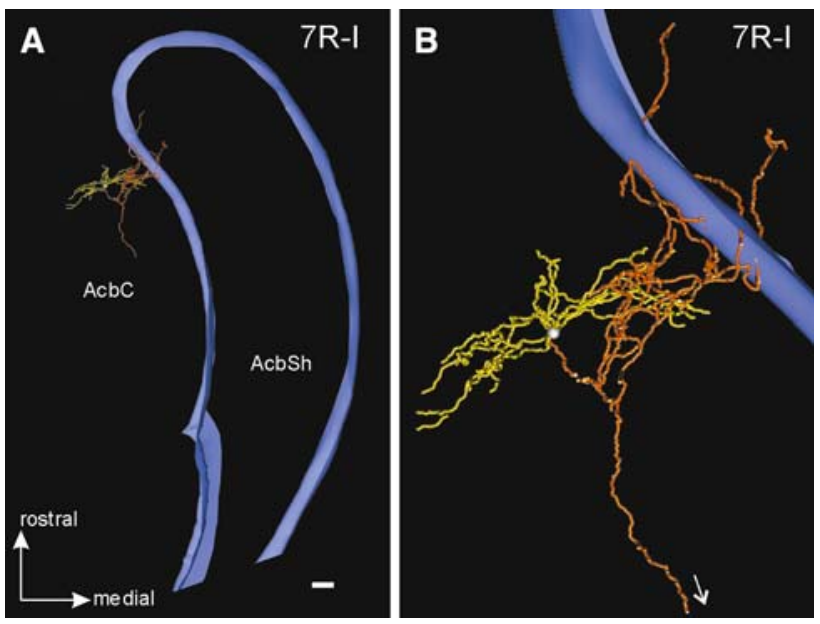

Fig. 13 Three-dimensional (3D) reconstruction of a single MSN in the Acb core subregion with local axon collaterals that cross the shellcore boundary. a The neuron (neuron 7R-I) is located in the rostromedial part of the core subregion of the Acb. In blue is indicated the contour of the Acb shell. Note that some of the local axon collaterals cross the shell-core boundary and extend into the rostral pole. b A zoomed view of the 3D-reconstruction of the neuron shown in a. The arrow indicates the main axon leaving the Acb. Abbreviations: $A c b C$ nucleus accumbens core, $A c b S h$ nucleus accumbens shell. Scale bar is $100 \mu \mathrm{m}$

Kawaguchi et al. 1989; Yelnik et al. 1994; Onn et al. 1994) and ventral striatum (Arts and Groenewegen 1992), that the geometry of the dendritic arborizations of MSN is influenced or limited by the borders between striatal 


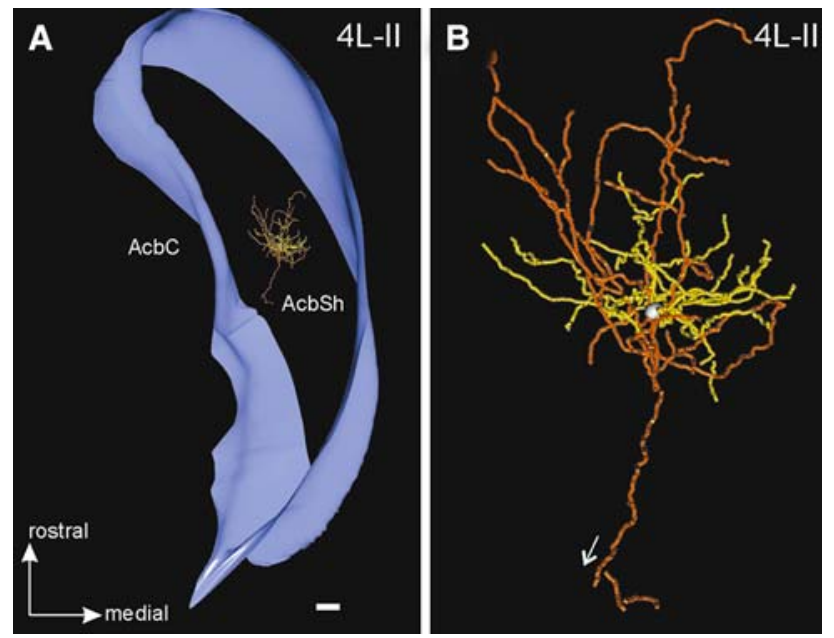

Fig. 14 Three-dimensional (3D) reconstruction of a single MSN in the Acb shell subregion with a rostralward extension of local axon collaterals and partial overlap between its dendrites and recurrent axon collaterals. a The neuron (neuron 4L-II) is located in the rostromedial part of the shell subregion of the Acb. In blue is indicated the contour of the Acb shell. Note that the axodendritic morphology (in yellow the dendrites and in orange the local axon collaterals) shows a partial overlap between the dendritic and axonal field and in addition a rostralward extension of some of its local axon collaterals. b A zoomed view of the 3D-reconstruction of the neuron shown in a. The arrow indicates the main axon leaving the Acb. Abbreviations: $A c b C$ nucleus accumbens core, AcbSh nucleus accumbens shell. Scale bar is $100 \mu \mathrm{m}$

compartments, i.e., the boundaries between patch and matrix. Our present observations in the core of the Acb confirm these findings, indicating that the dendrites of MSN remain within the compartment in which their parent cell body is located. This neuronal architecture supports the generally held view, primarily based on immunohistochemical and connectional data, that the patch and matrix form two largely segregated information processing compartments within the striatum (for reviews see Graybiel 1990; Gerfen 2004). However, the present study extends these observations by showing that MSN in either of these two larger subregions of the Acb, i.e., the shell and core, likewise retain their dendrites within the subregion in which the cell body resides. Like the dendrites of neuronal cell bodies that lie close to the border between patch and matrix, the dendrites of MSN in close proximity of the shell-core boundary tend to run in parallel to or are oriented away from this boundary. For neurons situated close to the boundary, this results in a very asymmetric and in some cases strongly flattened shape of the dendritic arborizations. The fact that the dendrites of MSN in shell or core do not cross the border between these two subregions of the Acb supports the notion that the shell and core constitute two morphologically and functionally largely segregated 'compartments' of the Acb. The distinction between shell and core on the basis of (immuno)histochemical and connectional criteria has been described extensively since its recognition by Zaborszky and colleagues (1985; for reviews, see Groenewegen et al. 1996, 1999; Zahm 1999). Likewise, the functional differentiation between shell and core has received extensive coverage in the literature (for reviews, see Cardinal et al. 2002; Di Chiara 2002; Kelley 2004). Our present findings suggest that it is unlikely that MSN in either Acb subregion are able to pick up information from incoming fibers in the adjacent subregion. This supports the idea of parallel and independent processing of information in both the shell and the core to be transmitted to their respective segregated target areas in the basal forebrain, diencephalon and mesencephalon (Groenewegen et al. 1996, 1999). As will be discussed below, recurrent axon collaterals of MSN in either core or shell might cross the border between the two subregions or the compartments therein and yet in this way providing a way of mutual influences at the striatal level (see also Van Dongen et al. 2005).

Apart from the direct influence of compartmental or subregional boundaries on the orientation and geometry of the dendritic arborizations of MSN that are situated in close proximity to such boundaries, the 3D reconstructions of most of the injected MSN in the present study show that the dendritic fields of MSN are flattened and that they show a preferred orientation. In the shell subregion, more than half of the neurons have a dendritic field that is preferentially oriented from rostral-dorsal-medial to caudal-ventral-lateral. Another, less numerous population of MSN shows a preferred orientation from dorsolateral to ventromedial. In the core the preferred orientations appear to be more heterogeneous with about one-third having an orientation from dorsomedial to ventrolateral, one-third from dorsolateral to ventromedial and the remaining neurons with a variety of orientations. It must be emphasized that the majority of neurons we injected in the shell are located in the medial part. In this part of the shell the afferent fibers from the ventral hippocampus enter, through the lateral septum, from a dorsal and medial position to run in ventral, lateral and rostral directions (Groenewegen et al. 1987). The amygdaloid afferents from the magnocellar basal amygdaloid nucleus enter the medial shell, through the bed nucleus of the stria terminalis, from caudal, medial and dorsal and run in rostral, lateral and ventral directions (Wright et al. 1996). Afferents from the ventral prelimbic and infralimbic areas enter the medial shell from rostral and run in caudal direction oriented more or less in a dorsal-medial to ventral-lateral direction (Berendse et al. 1992). In other words, the preferred orientation of the dendritic arborizations is more or less in parallel with the main afferent inputs of the medial shell of the Acb. This might be interpreted such that the dendrites of these neurons pick up information from a relatively restricted set of afferent fibers that make a 
maximal traverse through the dendritic arbors of individual neurons. Such an arrangement may lead to more contacts from a restricted afferent source, i.e., a form of convergence, as compared to the situation for the smaller population of MSN with a preferred dendritic arborization from dorsolateral to ventromedial. These dendritic fields tend to be oriented more or less perpendicular to the main stream of incoming fibers, and such an arrangement could result in fewer contacts with individual neurons but also with a larger number of neurons, i.e., a form of divergence. Convergence of inputs from different afferent sources onto single MSN in the shell of the Acb has been shown with both anatomical and electrophysiological methods for hippocampal and amygdaloid inputs (Mulder et al. 1998; French and Totterdell 2003) and anatomically for hippocampal and prefrontal fibers (French and Totterdell 2002). Whether the earlier mentioned differences in arrangement of incoming fibers versus orientations of dendritic arborizations make a real difference with respect to the number of contacts being made by single axons from hippocampal or amygdaloid origin with individual MSN in the medial shell remains to be established.

Within the core of the Acb the preferred orientations of dendritic arborizations of MSN show more variations. This might well be related to the less ordered input into this part of the ventral striatum and the complex 3D curvature of the shell-core boundary. Thus, although more dorsal parts of the core merge with the caudate-putamen complex and contains the typical patch-matrix structure and orientation of the dorsal striatum, the more ventral, lateral and rostral parts of the core are closely associated with the ventral and lateral shell, and the rostral shell (rostral pole) that curve around the core subregion. Although the orientation of afferent fiber systems in the caudate-putamen complex and the dorsal core is from dorsomedial to ventrolateral (cf. Berendse and Groenewegen 1990; Berendse et al. 1992; Wright et al. 1996; Voorn et al. 2004), the orientation of afferents to the ventral and rostral parts of the core is less well ordered.

In the head of the primate caudate nucleus, Walker et al. (1993) have shown that there exists a predominant dorsomedial to ventrolateral orientation of the dendritic arborizations of MSN, an orientation that parallels the main orientation of the striosomes in this part of the striatum. Desban et al. (1993) have demonstrated that the architecture of the patch network in the rat caudate-putamen likewise exhibits an overall dorsomedial to ventrolateral orientation. However, our results reveal that the preferred dendritic orientation of only a minority of the MSN in the Acb core conforms to this orientation. This might well be related to a somewhat more complex spatial orientation of the compartments and, as discussed above, a less ordered organization of afferents in this part of the striatum.
Relationships of MSN recurrent axon collaterals with subregional and compartmental boundaries and with the dendritic arborizations

While there are quite some studies describing the morphology of the dendrites and the 3D-geometry of the dendritic arborizations of MSN in the striatum, it has clearly been more difficult to fill the very delicate recurrent collateral, intrastriatal axonal ramifications of these neurons. In the present study, in slightly more than half of the neurons that were juxtacellularly injected, both dendrites and axons were sufficiently filled to allow adequate visualization of these fine intrastriatal axons. For the majority of these neurons the main axon exiting the striatum and terminating in the ventral pallidum or ventral mesencephalon could be identified (see also Van Dongen et al. 2005), indicating the adequacy of the labeling procedure. The present study reveals two interesting aspects concerning the recurrent axon collateral network of MSN in the ventral striatum. First, in contrast to the dendrites of MSN, recurrent axon collaterals of these neurons may cross subregional (shell-core) or compartmental (patch-matrix) boundaries in the ventral striatum and in this way interconnect these functionally different subregions or compartments. Indications for patch-matrix communication on the basis of axon collaterals of the MSN projection neurons have been very sparse until now. Kawaguchi et al. (1989), on the basis of intracellular injections of biocytin in in vitro slices of the dorsal striatum, concluded that both dendrites and axons of MSN respect the boundaries between patch and matrix. While Onn et al. (1994), using in vivo intracellular labeling, describe main projection axons of MSN located in the matrix of the dorsal striatum that cross patches on their way to the pallidum, these authors explicitly state that these axons do not show terminations in these patches. In a previous study from this laboratory, using intracellular injections of Lucifer Yellow in lightly fixed slices of the ventral striatum, a single MSN in the matrix could be identified that had extensive axon collaterals in the adjacent patch compartment (Arts and Groenewegen 1992). The results of the present study confirm these observations in showing that in the core of the Acb axons of labeled MSN may enter and terminate in a nearby patch. It must be emphasized, however, that intercompartmental connections on the basis of MSN projections are scarce and intercompartmental connections might be more prominent for interneurons with long axon collaterals, such as cholinergic (Meredith et al. 1989; Kawaguchi 1993) and somatostatin interneurons (Chesselet and Graybiel 1986). The axons of the large aspiny cholinergic interneurons, however, tend to respect compartmental boundaries (Meredith et al. 1989; Kawaguchi 1993). This has been established most directly with 
intracellular labeling of individual neurons in the dorsal striatum (Kawaguchi 1993). With immunohistochemical studies, Chesselet and Graybiel (1986) have demonstrated that somatostatin interneurons might cross compartmental boundaries. The parvalbumin and calretinin interneurons may also be involved in patch-matrix intercommunication, as demonstrated by immunohistochemical studies (Cowan et al. 1990; Fortin and Parent 1994). As discussed previously (Van Dongen et al. 2005), MSN recurrent axon collaterals might also be the substrate for communication between shell and core of the Acb. Although these projections are bidirectional, as also shown in the present study, there appears to be dominance for core-to-shell projections when the results of larger tracer injections are taken into account.

The second interesting aspect revealed in the present study concerns the distribution of the recurrent axonal network in relationship to the dendritic arborizations of the same striatal projection neuron. Principally, three patterns were distinguished: (1) MSN with a distribution of axon collaterals largely falling within the space of the dendritic distribution ('full overlap'); (2) MSN with axonal collaterals extending beyond the reaches of the dendritic arborizations, but with a considerable degree of overlap ('partial overlap'); (3) MSN with recurrent axon collaterals occupying a 3D-space that showed no or only minimal overlap with the dendrites of the same neuron (no overlap). In the shell subregion, all injected neurons belonged to the second category, indicating that the axon collaterals of MSN in this part of the Acb in principle reach other shell neurons primarily within but also beyond the 'receptive' sphere of their dendrites. In the core subregion, neurons of all three categories were identified. One neuron with no overlap between dendrites and axon collaterals exhibited a widely distributed axonal network extending up to $1 \mathrm{~mm}$ away from the parent cell body. In particular, the findings in the core are largely in agreement with those of earlier studies in the dorsal striatum. Both Bishop et al. (1982) and Kawaguchi et al. (1990) distinguished two categories of striatal projection neurons. One category consists of neurons with axons that are largely overlapping the dendritic field of the same neuron. Within this category several subtypes were described on the basis of their extrinsic projections to pallidal and mesencephalic targets. A second category consists of neurons with axons that have a wide distribution of their axon collaterals, up to 1-2 $\mathrm{mm}$ within the striatum, and that do not project beyond the pallidum (Kawaguchi et al. 1990). Like in our sample, neurons in the latter category were far less numerous than MSN with short-range recurrent axonal fields. Whether MSN with longer ranging axon collaterals exist within the Acb shell remains uncertain; the present sample of juxtacellulary filled neurons does not include neurons of that category.
Nevertheless, it has been suggested on the basis of 'classical' small tracer injections that the shell has more extensive and wide-ranging associative intrastriatal projections than the core and the rest of the striatum (Heimer et al. 1991; Van Dongen et al. 2005). However, whether such long-range intrastriatal projections are established by recurrent collaterals from MSN or axons of interneurons cannot be concluded on the basis of the present results.

Intrastriatal communication: interactions between MSN?

Medium-sized spiny projection neurons are the most prevalent neuronal cell type in both the dorsal and ventral striatum, by far outnumbering the smaller and heterogeneous population of striatal interneurons. MSN with their extensive 'receptive' surface in the form of dendrites and spines, represent the neuronal substrate par excellence for the integration of information from functionally different cortical areas, limbic structures, and midline and intralaminar thalamic nuclei in the striatum. These inputs arrive mainly on the heads of the dendritic spines of MSN while the transfer of this information, that is excitatory in nature, can be modulated by dopaminergic inputs and contacts from striatal interneurons. Dopaminergic fibers make contact on the neck of the spines or on the dendritic shafts while the terminals of cholinergic and GABAergic interneurons are positioned more proximally on dendritic shafts and the perikaryon of the MSN (reviews, e.g., Smith and Bolam 1990; Gerfen 2004; Tepper et al. 2004). As discussed above, it has long been known that MSN have an extensive local recurrent collateral axonal network (Ramón y Cajal 1911; Preston et al. 1980; Bishop et al. 1982; Chang and Kitai 1985) and on the basis of ultrastructural studies, it has been demonstrated that these axons make inhibitory synaptic contacts on the dendritic shafts of neighboring MSN (e.g., Bolam and Izzo 1988; Meredith et al. 1993; Tunstall et al. 2002). Thus, the inhibitory inputs from spatially closely related MSN appear to be in a strategic position on the dendrites of an MSN to influence the transfer of information from the dendrites to the cell body. While it has been notoriously difficult to show functional synaptic contacts between MSN with electrophysiological methods (e.g., Jaeger et al. 1994; see also Tepper et al. 2004), several recent studies have confirmed their functional existence in both the dorsal (Tunstall et al. 2002; Czubayko and Plenz 2002; Venance et al. 2004) and ventral striatum (Taverna et al. 2004). Taverna et al. (2004), using dual whole cell patch-clamp recordings in acute slices of the Acb, showed that no less than $34 \%$ of the patched pairs of MSN had synaptic connections, most of them uni-directionally. It could further be shown that the 
connections between MSN exhibit various forms of shortterm synaptic plasticity and that the functional connectivity can be modulated by dopamine (Taverna et al. 2004, 2005; also Czubayko and Plenz 2002).

Striatal interneurons, including the fast-spiking GABAergic interneurons, are known to contact MSN and play an important role in modulating their output (for review see Tepper et al. 2004). There are no electrophysiological data about possible connections from MSN to these interneurons. Theoretically such connections would have an influence over a wider striatal area than the interconnections between MSN because interneurons have a much more extensive axonal network than MSN. However, because little is known about MSN to interneuron projections, we concentrate our further discussion on the possible functional aspects of interactions between MSN.

In theories of striatal functioning, synaptic connections between MSN have been interpreted as being important for striatal learning and selection mechanisms through lateral inhibitory processes. Lateral inhibition between MSN is thought to play a role in the interactions between collections or 'ensembles' of neurons that are influenced by different sets of cortical inputs and code for competing outputs of the striatum (e.g., Pennartz et al. 1994; Plenz 2003; Tepper et al. 2004). In this way, lateral inhibition between MSN would support a 'winner take all' mechanism in the selection of striatal outputs. Recent insights into the role of an important class of inhibitory striatal interneurons, i.e., the fast-spiking GABAergic interneurons that are driven by excitatory cortical inputs and have a much more powerful inhibitory influence on MSN than recurrent collaterals of MSN themselves, have again questioned the significance of MSN-MSN interactions (Tepper et al. 2004). However, as argued by Plenz (2003), the inhibitory mechanisms of fast-spiking interneurons and collateral connections between MSN could both play a distinctive role in the striatal network dynamics that underlie timing and selection of cortical information that is processed through the basal ganglia circuitry. Thus, even though the direct electrophysiological effect of MSN inputs on adjacent neurons might not be as strong, the consistent findings that these MSN-MSN connections exhibit several forms of short-term plasticity and are influenced by dopamine suggests a role for such interactions at the striatal microcircuit level (Taverna et al. 2004, 2005; also Czubayko and Plenz 2002), a role that has to be still further explored.

In further considering the functional role of connections between MSN, the results of the present study with respect to the distribution of recurrent axon collaterals of MSN in the ventral striatum should be taken into account. First, most theoretical models depart from the assumption that the geometry of the axonal arborizations of MSN largely concurs with the dendritic geometry of the parent cell body. Our study, in agreement with Kawaguchi et al. (1990), shows that such congruence is not always the case and that there exist MSN with long-range axonal terminations and several forms of nonoverlapping axonal and dendritic arborizations. This could imply that such recurrent axon collaterals connect functionally different sets of MSN and provide a way of interaction or competition between such populations of neurons. Important in this context is the demonstration of directionality in the intrastriatal connections as described in our previous study (Van Dongen et al. 2005) and confirmed in the present study at the single cell level. Second, our findings of recurrent axon collaterals of MSN that cross the patch-matrix and the shell-core borders emphasize and strengthen the idea that such collaterals play a role in the interaction between functionally different compartments or 'ensembles' of neurons (cf also Pennartz et al. 1994; Van Dongen et al. 2005).

Open Access This article is distributed under the terms of the Creative Commons Attribution Noncommercial License which permits any noncommercial use, distribution, and reproduction in any medium, provided the original author(s) and source are credited.

\section{References}

Arts MP, Groenewegen HJ (1992) Relationships of the dendritic arborizations of ventral striatomesencephalic projection neurons with boundaries of striatal compartments. An in vitro intracellular labelling study in the rat. Eur J Neurosci 4:574-588

Berendse HW, Groenewegen HJ (1990) Organization of the thalamostriatal projections in the rat, with special emphasis on the ventral striatum. J Comp Neurol 299:187-228

Berendse HW, Galis-de Graaf Y, Groenewegen HJ (1992) Topographical organization and relationship with ventral striatal compartments of prefrontal corticostriatal projections in the rat. $\mathbf{J}$ Comp Neurol 316:314-347

Bishop GA, Chang HT, Kitai ST (1982) Morphological and physiological properties of neostriatal neurons: an intracellular horseradish peroxidase study in the rat. Neurosci 7:179-191

Bolam JP, Izzo PN (1988) The postsynaptic targets of substance Pimmunoreactive terminals in the rat neostriatum with particular reference to identified spiny striatonigral neurons. Exp Brain Res 70:361-377

Bolam JP, Izzo PN, Graybiel AM (1988) Cellular substrate of the histochemically defined striosome/matrix system of the caudate nucleus: a combined Golgi and immunocytochemical study in cat and ferret. Neurosci 24:853-875

Cardinal RN, Parkinson JA, Hall J, Everitt BJ (2002) Emotion and motivation: the role of the amygdala, ventral striatum, and prefrontal cortex. Neurosci Biobehav Rev 26:321-352

Chang HT, Kitai ST (1985) Projection neurons of the nucleus accumbens: an intracellular labeling study. Brain Res 347:112116

Chesselet MF, Graybiel AM (1986) Striatal neurons expressing somatostatin-like immunoreactivity: evidence for a peptidergic interneuronal system in the cat. Neurosci 17:547-571

Cowan RL, Wilson CJ, Emson PC, Heizmann CW (1990) Parvalbumin-containing GABAergic interneurons in the rat neostriatum. J Comp Neurol 302:197-205 
Czubayko U, Plenz D (2002) Fast synaptic transmission between striatal spiny projection neurons. Proc Natl Acad Sci 99:1576415769

DeFelipe J, Alonso-Nanclares L, Arellano JI (2002) Microstructure of the neocortex: comparative aspects. J Neurocytol 31:337-346

Desban M, Kemel ML, Glowinski J, Gauchy C (1993) Spatial organization of patch and matrix compartments in the rat striatum. Neurosci 57:661-671

Di Chiara G (2002) Nucleus accumbens shell and core dopamine: differential role in behavior and addiction. Behav Brain Res 137:75-114

Feldman ML (1984) Morphology of the neocortical pyramidal neuron. In: Peters A, Jones EG (eds) Cerebral cortex, vol 1, cellular components of the cerebral cortex. Plenum, New York, pp 123-200

Fortin M, Parent A (1994) Patches in the striatum of squirrel monkeys are enriched with calretinin fibers but devoid of calretinin cell bodies. Neurosci Lett 182:51-54

French SJ, Totterdell S (2002) Hippocampal and prefrontal cortical inputs monosynaptically converge with individual projection neurons of the nucleus accumbens. J Comp Neurol 446:151-165

French SJ, Totterdell S (2003) Individual nucleus accumbensprojection neurons receive both basolateral amygdala and ventral subicular afferents in rats. Neuroscience 119:19-31

Gerfen CR (1985) The neostriatal mosaic. I. Compartmental organization of projections from the striatum to the substantia nigra in the rat. J Comp Neurol 236:454-476

Gerfen CR (2004) Basal ganglia. In: Paxinos G (ed) The rat nervous system, 3rd edn. Elsevier Academic Press, San Diego, pp 455-508

Gray EG (1959) Axosomatic and axodendritic synapses of the cerebral cortex: an electron microscope study. J Anat 93:420 433

Graybiel AM (1990) Neurotransmitters and neuromodulators in the basal ganglia. Trends Neurosci 13:244-254

Groenewegen HJ, Vermeulen-Van der Zee E, te Kortschot A, Witter MP (1987) Organization of the projections from the subiculum to the ventral striatum in the rat. A study using anterograde transport of Phaseolus vulgaris leucoagglutinin. Neurosci 23:102-120

Groenewegen HJ, Wright CI, Beijer AVJ (1996) The nucleus accumbens: gateway for limbic structures to reach the motor system? Prog Brain Res 107:485-511

Groenewegen HJ, Wright CI, Beijer AVJ, Voorn P (1999) Convergence and segregation of ventral striatal inputs and outputs. In: Advancing from the ventral striatum to the extended amygdala: implications for neuropsychiatry and drug abuse. Proc N Y Acad Sci 877:49-63

Heimer L, Zahm DS, Churchill L, Kalivas PW, Wohltmann C (1991) Specificity in the projection patterns of accumbal core and shell in the rat. Neurosci 41:89-125

Jaeger D, Kita H, Wilson CJ (1994) Surround inhibition among projection neurons is weak or nonexistent in the rat neostriatum. J Neurophysiol 72:2555-2558

Jongen-Rêlo AL, Groenewegen HJ, Voorn P (1993) Evidence for a multi-compartmental histochemical organization of the nucleus accumbens in the rat. J Comp Neurol 337:267-276

Jongen-Rêlo AL, Voorn P, Groenewegen HJ (1994) Immunohistochemical characterization of the shell and core territories of the nucleus accumbens in the rat. Eur J Neurosci 6:1255-1264

Kawaguchi Y (1993) Physiological, morphological, and histochemical characterization of three classes of interneurons in rat neostriatum. J Neurosci 13:4908-4923

Kawaguchi Y, Wilson CJ, Emson PC (1989) Intracellular recording of identified neostriatal patch and matrix spiny cells in a slice preparation preserving cortical inputs. J Neurophys 65:10521068
Kawaguchi Y, Wilson CJ, Emson PC (1990) Projection subtypes of rat neostriatal matrix cells revealed by intracellular injection of biocytin. J Neurosci 10:3421-3438

Kelley AE (2004) Ventral striatal control of appetitive motivation: role in ingestive behavior and reward-related learning. Neurosci Biobehav Rev 27:765-776

Mahon D, Deniau JM, Charpier S (2001) Relationship between EEG potentials and intracellular activity of striatal and cortico-striatal neurons: an in vivo study under different anesthetics. Cereb Cortex 11:360-374

Mallet N, Le Moine C, Charpier S, Gonon F (2005) Feedforward inhibition of projection neurons by fast-spiking GABA interneurons in the rat striatum in vivo. J Neurosci 25:3857-3869

Meredith GE, Blank B, Groenewegen HJ (1989) The distribution and compartmental organization of the cholinergic neurons in nucleus accumbens of the rat. Neurosci 31:327-345

Meredith GE, Agolia R, Arts MP, Groenewegen HJ, Zahm DS (1992) Morphological differences between projection neurons of the core and shell in the nucleus accumbens of the rat. Neuroscience 50:149-162

Meredith GE, Ingham CA, Voorn P, Arbuthnott GW (1993) Ultrastructural characteristics of enkephalin-immunoreactive boutons and their postsynaptic targets in the shell and core of the nucleus accumbens of the rat. J Comp Neurol 332:224-236

Mulder AB, Hodenpijl MG, Lopes da Silva FH (1998) Electrophysiology of the hippocampal and amygdaloid projections to the nucleus accumbens of the rat: convergence, segregation, and interaction of inputs. J Neurosci 18:5095-5102

Onn SP, Berger TW, Grace AA (1994) Identification and characterization of striatal cell subtypes using in vivo intracellular recording and dye-labeling in rats: III. Morphological correlates and compartmental localization. Synapse 16:231-254

Paxinos G, Watson C (1986) The rat brain in stereotaxic coordinates. Academic, New York

Pennartz CMA, Groenewegen HJ, Lopes Da Silva FH (1994) The nucleus accumbens as a complex of functionally distinct neuronal ensembles: an integration of behavioural, electrophysiological and anatomical data. Progr Neurobiol 42:719-761

Penny GR, Wilson CJ, Kitai ST (1988) Relationship of the axonal and dendritic geometry of spiny projection neurons to the compartmental organization of the neostriatum. J Comp Neurol 269:275289

Pinault D (1996) Golgi-like labeling of a single neuron recorded extracellularly. Neurosci Lett 170:255-260

Plenz D (2003) When inhibition goes incognito: feedback interaction between spiny projection neurons in striatal function. Trends Neurosci 26:436-443

Preston RJ, Bishop GA, Kitai ST (1980) Medium spiny neurons projection from the rat striatum: an intracellular horseradish peroxidase study. Brain Res 185:253-263

Ramón y Cajal S (1911) Histologie du Systeme Nerveaux de l'Hommes et de Vertébrés. In : Maloine A (ed), Paris

Slaght SJ, Paz T, Chavez M, Deniau JM, Mahon S, Charpier S (2004) On the activity of the corticostriatal networks during spike-andwave discharges in a genetic model of absence epilepsy. J Neurosci 24:6816-6825

Smith AD, Bolam JP (1990) The neural network of the basal ganglia as revealed by the study of synaptic connections of identified neurones. Trends Neurosci 13:259-265

Taverna S, van Dongen YC, Groenewegen HJ, Pennartz CMA (2004) Direct physiological evidence for synaptic connectivity between medium-sized spiny neurons in rat nucleus accumbens in situ. $\mathbf{J}$ Neurophysiol 91:1111-1121

Taverna S, Canciani B, Pennartz CMA (2005) Dopamine D1receptors modulate lateral inhibition between principal cells of the nucleus accumbens. J Neurophysiol 93:1816-1819 
Tepper JM, Koos T, Wilson CJ (2004) GABAergic microcircuits in the neostriatum. Trends Neurosci 27:662-669

Tunstall MJ, Oorschot DE, Kean A, Wickens JR (2002) Inhibitory interactions between spiny projection neurons in the rat striatum. J Neurophysiol 88:1263-1269

Van Dongen YC, Deniau JM, Pennartz CMA, Galis-de Graaf Y, Voorn P, Thierry AM, Groenewegen HJ (2005) Anatomical evidence for direct connections between the shell and core subregions of the rat nucleus accumbens. Neurosci 136:10491071

Veenman CL, Reiner A, Honig MG (1992) Biotinylated dextran amine as an anterograde tracer for single- and double-labeling studies. J Neurosci Methods 41:239-254

Venance L, Glowinski J, Gaiume C (2004) Electrical and chemical transmission between striatal GABAergic output neurons in rat brain slices. J Physiol 559:215-230

Voorn P, Gerfen CR, Groenewegen HJ (1989) Compartmental organization of the ventral striatum of the rat: immunohistochemical distribution of enkephalin, substance $\mathrm{P}$, dopamine, and calcium-binding protein. J Comp Neurol 289:189-201

Voorn P, Vanderschuren LJ, Groenewegen HJ, Robbins TW, Pennartz CM (2004) Putting a spin on the dorsal-ventral divide of the striatum. Trends Neurosci 27:468-474
Walker RH, Graybiel AM (1993) Dendritic arbors of spiny neurons in the primate striatum are directionally polarized. J Comp Neurol 337:629-639

Wilson CJ (1993) The generation of natural firing patterns in neostriatal neurons. Prog Brain Res 99:277-297

Wright CI, Groenewegen HJ (1996) Patterns of overlap and segregation between insular cortical, intermediodorsal thalamic and basal amygdaloid afferents in the nucleus accumbens of the rat. Neurosci 73:359-373

Yelnik J, Francois C, Percheron G (1994) Three-dimensional morphology of striatal neurons in relation to compartmental organization of the striatum. In: Percheron G (ed) The Basal Ganglia IV. Plenum, New York, pp 43-49

Zaborszky L, Alheid GF, Beinfeld MC, Eiden LE, Heimer L, Palkovits M (1985) Cholecystokinin innervation of the ventral striatum: a morphological and radioimmunological study. Neurosci 4:427-453

Zahm DS (1999) Functional-anatomical implications of the nucleus accumbens core and shell subterritories. Ann N Y Acad Sci 877:113-128

Zahm DS, Brog JS (1992) On the significance of subterritories in the "accumbens" part of the rat ventral striatum. Neurosci 50:751-767 